Working Paper/Document de travail 2012-34

\title{
The Economic Value of Realized Volatility: Using High-Frequency Returns for Option Valuation
}

by Peter Christoffersen, Bruno Feunou, Kris Jacobs and Nour Meddahi 
Bank of Canada Working Paper 2012-34

October 2012

\title{
The Economic Value of Realized Volatility: Using High-Frequency Returns for Option Valuation
}

by

\author{
Peter Christoffersen, ${ }^{1}$ Bruno Feunou, ${ }^{2}$ Kris Jacobs ${ }^{3}$ and \\ Nour Meddahi ${ }^{4}$ \\ 1Rotman School of Management \\ University of Toronto \\ Toronto, Ontario, Canada M5S 3E6 \\ Address correspondence to: peter.christoffersen@rotman.utoronto.ca \\ 2Financial Markets Department \\ Bank of Canada \\ Ottawa, Ontario, Canada K1A 0G9 \\ 3University of Houston \\ ${ }^{4}$ Toulouse School of Economics
}

Bank of Canada working papers are theoretical or empirical works-in-progress on subjects in economics and finance. The views expressed in this paper are those of the authors. No responsibility for them should be attributed to the Bank of Canada. 


\section{Acknowledgements}

For helpful comments we would like to thank seminar participants at the Bank of Canada, the Econometric Society World Congress, the Society for Financial Econometrics, and the Toulouse School of Economics. Christoffersen is also affiliated with the Copenhagen Business School and CREATES and acknowledges financial support from SSHRC. 


\begin{abstract}
Many studies have documented that daily realized volatility estimates based on intraday returns provide volatility forecasts that are superior to forecasts constructed from daily returns only. We investigate whether these forecasting improvements translate into economic value added. To do so we develop a new class of affine discrete-time option valuation models that use daily returns as well as realized volatility. We derive convenient closed-form option valuation formulas and we assess the option valuation properties using S\&P500 return and option data. We find that realized volatility reduces the pricing errors of the benchmark model significantly across moneyness, maturity and volatility levels.
\end{abstract}

JEL classification: G13

Bank classification: Asset pricing; Econometric and statistical methods

\title{
Résumé
}

De nombreuses études ont montré que les estimations de la volatilité réalisée quotidienne qui se fondent sur les rendements intrajournaliers permettent d'aboutir à des prévisions de la volatilité plus justes que les projections faites uniquement à partir des rendements quotidiens. Les auteurs cherchent à déterminer si une telle amélioration prévisionnelle se traduit par une plus-value économique. À cette fin, ils construisent une nouvelle classe de modèles affines en temps discret d'évaluation des options qui font appel aux rendements quotidiens et également à la volatilité réalisée. Ils déduisent des formules analytiques des prix d'options et comparent, à l'aide de données sur les rendements et options liés à l'indice S\&P500, les propriétés des modèles à l'étude. Ils constatent que la volatilité réalisée réduit de manière significative les erreurs d'évaluation du modèle de référence pour tous les degrés de parité, échéances et niveaux de volatilité examinés.

Classification JEL : G13

Classification de la Banque : Évaluation des actifs; Méthodes économétriques et statistiques 


\section{Introduction}

ARCH models (Engle, 1982) and their extensions (Bollerslev, 1986, Nelson, 1991, Glosten, Jagannathan, and Runkle, 1993) have proven very successful for describing the time series behavior of conditional variances of financial asset returns (French, Schwert and Stambaugh, 1987). Statistical tools including the likelihood principle strongly favor ARCH and GARCH over models with constant variance, and the models have therefore found widespread use in finance to model stock returns, interest rates, exchange rates, and option prices.

One important criticism of GARCH models concerns their apparent shortcomings in forecasting volatility, as measured by the $R^{2}$ of a Mincer-Zarnowitz regression that uses squared daily return as a proxy for ex-post variance on the left-hand side and the ex-ante GARCH forecast of volatility on the right-hand side. Andersen and Bollerslev (1998) make two important contributions in this regard. First, they prove theoretically and show in simulations that when the GARCH model is the true data generating process, the $R^{2} \mathrm{~s}$ of Mincer-Zarnowitz regressions can be expected to be low, and are in fact of similar magnitude as the empirically observed $R^{2}$ s. Andersen and Bollerslev (1998) note that this apparent lack of predictive ability is due to the use of the squared daily returns as the ex-post variance proxy in the regression, because the squared daily return is a very noisy measure. Andersen and Bollerslev's second contribution is to demonstrate that realized volatility, measured as the sum of squared intra-daily returns, is a superior measure of ex-post variance, and leads to much higher $\mathrm{R}^{2} \mathrm{~s}$ in the Mincer-Zarnowitz regression using the same ex-ante GARCH variance forecasts.

Following the realization that accurate measures of volatility can be obtained from high frequency data, a growing literature has developed that studies the properties of realized volatility. Andersen, Bollerslev, Diebold, and Labys (2003) propose time series models for realized volatility in order to more accurately predict volatility. Joint models for returns and realized volatility have been proposed, either ignoring the contribution of jumps (Forsberg and Bollerslev, 2002) or by incorporating them in the model (Bollerslev, Kretschmer, Pigorsch, and Tauchen, 2009). ${ }^{1}$

A recent literature develops GARCH-style models that incorporate the information from realized volatility (see Chen, Ghysels, and Wang, 2011, Hansen, Huang, and Shek, 2011, and Shephard and Sheppard, 2010). Several of the models in this literature can be cast in the multiple indicators framework of Engle and Gallo (2006). The basic idea to include realized volatility on the righthand-side of a GARCH model dates to Engle (2002).

\footnotetext{
${ }^{1}$ Other studies investigate the properties of realized volatility when the sampling interval for intra-day returns converges to zero (e.g., Barndorff-Nielsen and Shephard, 2002), when there are market frictions (e.g., Zhang, Mykland, and Aït-Sahalia, 2005), and when one faces different types of jumps (see e.g., Aït-Sahalia and Jacod, 2009, and the references therein).
} 
A few authors jointly model returns and realized volatility for the purpose of option pricing. Following the density modeling approach in Forsberg and Bollerslev (2002), Stentoft (2008) assumes that the conditional distribution of realized volatility is Inverse Gaussian with time-varying mean, while returns are assumed to be conditionally normal with variance equal to current realized volatility. Corsi, Fusari, and La Vecchia (2009) follow a similar approach by jointly modeling returns and realized volatility. The models in these studies are not affine, and therefore pricing European options is done using simulation, making inference challenging. Moreover, the models are not estimated using options data. Estimation exclusively relies on returns and realized volatility, which limits these studies' ability to study risk premia.

We develop a new type of affine discrete-time models that allows for closed-form option valuation formulas using the conditional moment-generating function. We model daily returns as well as expected realized volatility. The volatility dynamic for the resulting models contains a GARCH component that consists of daily lagged squared returns, but also an expected realized volatility component. We refer to this model as the generalized affine realized volatility (GARV) model. The GARV model nests the daily Heston and Nandi (2000) GARCH model as a special case, and it also nests a pure realized variance model as a special case, which we refer to as the ARV model. We deliberately do not model the intraday dynamics of returns as they are dominated by market microstructure effects which are unlikely to affect the valuation of options with several months to maturity.

We implement and test our models using daily returns, realized volatility and options data. First, we estimate the models by optimizing the fit of S\&P500 returns and realized volatility using maximum likelihood. Second, we optimize the fit of S\&P500 index options using non-linear least squares. Third, we estimate the models by optimizing the joint fit of S\&P500 returns, realized volatility, and options, which necessitates the modeling of risk premia. In all three estimation exercises we filter volatility on returns and realized volatility. We find that incorporating past realized volatilities leads to a better fit on returns and realized volatility, and that it reduces the option pricing errors of the benchmark model significantly across moneyness, maturity and volatility levels. For all three estimation exercises, both GARCH and realized volatility help to model returns and options, and therefore the GARV model outperforms the GARCH model as well as the ARV model. When directly comparing the ARV model with the GARCH model, the ARV model performs better in all three estimation exercises. We demonstrate that the improved performance of our newly proposed models is due to their ability to more adequately model higher moments, in particular the volatility of variance.

The paper proceeds as follows. Section 2 introduces the new models. Section 3 presents model estimates obtained optimizing the fit on a long sample of returns and realized volatilities. Section 4 
develops the models' risk neutral distribution. Section 5 estimates the models fitting option prices using a large sample of contracts while filtering volatility on returns and realized volatility. Section 6 estimates the models jointly optimizing the fit of returns, realized volatility and options. Section 7 introduces two-component and non-affine alternative specifications and Section 8 concludes. Some of the more technical material is collected in appendices.

\section{Modeling Return Dynamics Using Realized Volatility}

This section builds a new affine dynamic model that employs the information embedded in daily realized volatility, while nesting the affine discrete time option pricing model of Heston and Nandi (2000), which we first describe.

\subsection{The Affine GARCH Model}

Heston and Nandi (2000) assume the following process for daily log returns

$$
R_{t+1} \equiv \ln \left(S_{t+1} / S_{t}\right)=r+\lambda h_{t}-\frac{1}{2} h_{t}+\sqrt{h_{t}} \varepsilon_{t+1}
$$

where $r$ denotes the risk-free rate, and $\lambda$ denotes the price of risk. ${ }^{2}$ The i.i.d. standard normal error term is represented by $\varepsilon_{t+1}$. The first two conditional moments of returns in the model are

$$
\begin{aligned}
E_{t}\left(R_{t+1}\right) & =r+\left(\lambda-\frac{1}{2}\right) h_{t} \\
\operatorname{Var}_{t}\left(R_{t+1}\right) & =h_{t}
\end{aligned}
$$

so that $h_{t}$ is the conditional variance for day $t+1$ which is known at the end of day $t .^{3}$ Note that the expected return is linear in the conditional variance.

The variance process takes the following form

$$
h_{t+1}=\omega_{1}+\beta_{1} h_{t}+\alpha_{1}\left(\varepsilon_{t+1}-\gamma_{1} \sqrt{h_{t}}\right)^{2},
$$

where $\gamma_{1}$ captures the asymmetric volatility response, often referred to as the leverage effect. We

\footnotetext{
${ }^{2}$ For alternative GARCH option pricing models, see Barone-Adesi, Engle, and Mancini (2008), Bollerslev and Mikkelsen (1999), Duan (1995), Engle and Mustafa (1992), and Ritchken and Trevor (1999).

${ }^{3}$ Note that to keep notation consistent with the realized volatility processes, our timing convention is a little different from Heston and Nandi (2000) and from the conventional GARCH notation. Furthermore, Heston and Nandi (2000) do not include the $-\frac{1}{2} h_{t}$ term, thus $\lambda=-\frac{1}{2}$ corresponds to risk-neutrality in their notation whereas $\lambda=0$ corresponds to risk-neutrality in ours.
} 
will refer to this model as GARCH below.

Before proceeding note that we can rewrite the GARCH model as follows

$$
h_{t+1}=\left(\omega_{1}+\alpha_{1}\right)+\left(\beta_{1}+\alpha_{1} \gamma_{1}^{2}\right) h_{t}+\alpha_{1} v\left(\varepsilon_{t+1}\right)
$$

where $\left(\beta_{1}+\alpha_{1} \gamma_{1}^{2}\right)$ is the persistence of daily variance and where $v\left(\varepsilon_{t+1}\right)$ is a zero-mean innovation defined by

$$
v\left(\varepsilon_{t+1}\right)=\left[\left(\varepsilon_{t+1}-\gamma_{1} \sqrt{h_{t}}\right)^{2}-\left(1+\gamma_{1}^{2} h_{t}\right)\right]
$$

Note also that from (2.2) it is easy to derive the unconditional variance to be

$$
E\left[h_{t}\right]=\frac{\left(\omega_{1}+\alpha_{1}\right)}{1-\left(\beta_{1}+\alpha_{1} \gamma_{1}^{2}\right)} .
$$

Note that unlike other GARCH models, $\omega_{1}$ can be zero in the Heston-Nandi model and the unconditional variance will still be positive, as long as $\alpha_{1}$ is positive and persistence is less than one.

The so-called leverage covariance and the variance of variance can be derived as

$$
\begin{aligned}
\operatorname{Cov}_{t}\left(R_{t+1}, h_{t+1}\right) & =-2 \alpha_{1} \gamma_{1} h_{t}, \text { and } \\
\operatorname{Var}_{t}\left(h_{t+1}\right) & =2 \alpha_{1}^{2}\left(1+2 \gamma_{1}^{2} h_{t}\right)
\end{aligned}
$$

which unlike other GARCH models are linear in the conditional variance as well. We will use these moments to compare models in the empirical study below.

\subsection{The Generalized Affine RV (GARV) Model}

The seminal paper by Andersen, Bollerslev, Diebold and Labys (2003) contains the important intuition that realized volatility helps in forecasting future volatility, because it provides a better assessment of current spot volatility. GARCH models instead need to infer today's volatility from a moving average of past daily squared returns. This intuition motivates us to build an option valuation model where realized volatility is used to construct today's spot volatility. This should in turn lead to better estimates of the volatility term structure, and thus to more accurate option prices.

In order to use the model for option valuation, we ultimately need to derive the risk-neutral process and for this we first need a fully specified physical model. We will be using daily returns and daily RV as our two observed variables and we therefore need to fully specify their joint dynamics. 
Our ultimate goal is to value options in a fast and reliable fashion. We therefore focus on the affine class of models where the moment generating function can be derived.

We assume the following dynamic model for daily returns

$$
R_{t+1}=r+\lambda \bar{h}_{t}-\frac{1}{2} \bar{h}_{t}+\sqrt{\bar{h}_{t}} \varepsilon_{1, t+1}
$$

where $\varepsilon_{1, t+1}$ is a standard normal return shock and where the return moments are

$$
\begin{aligned}
E_{t}\left(R_{t+1}\right) & =r+\left(\lambda-\frac{1}{2}\right) \bar{h}_{t} \\
\operatorname{Var}_{t}\left(R_{t+1}\right) & =\bar{h}_{t}
\end{aligned}
$$

Return variance, $\bar{h}_{t}$, is a function of two components which we now define.

Just as in Heston and Nandi (2000), we will use the daily return shocks, $\varepsilon_{1, t+1}$, to form a return-based conditional variance component of the form

$$
h_{t+1}^{R}=\omega_{1}+\beta_{1} h_{t}^{R}+\alpha_{1}\left(\varepsilon_{1, t+1}-\gamma_{1} \sqrt{\bar{h}_{t}}\right)^{2}
$$

We now need to introduce a contribution to conditional variance from RV. Our approach is to develop a model in which the conditional expectation of RV has an affine GARCH form. We first assume that the expected RV has the following autoregressive structure in $\mathrm{RV}^{4}$

$$
E_{t}\left[R V_{t+1}\right] \equiv h_{t}^{R V}=\omega_{2}+\kappa \alpha_{2} \beta_{2} \gamma_{2} h_{t-1}^{R}+\theta h_{t-1}^{R V}+\beta_{2} R V_{t}
$$

We next assume that the observation on $R V_{t+1}$ is linked to its standard normal innovation term $\varepsilon_{2, t+1}$ via

$$
R V_{t+1}=h_{t}^{R V}+\alpha_{2} v\left(\varepsilon_{2, t+1}\right)
$$

where $v\left(\varepsilon_{2, t+1}\right)$ is a zero-mean innovation defined by

$$
v\left(\varepsilon_{2, t+1}\right)=\left[\left(\varepsilon_{2, t+1}-\gamma_{2}{\sqrt{\bar{h}_{t}}}^{2}-\left(1+\gamma_{2}^{2} \bar{h}_{t}\right)\right] .\right.
$$

Note that we are modeling the expected RV instead of the raw RV thus effectively smoothing the RV process. By substituting (2.8) and (2.9) into (2.7) we can rewrite the conditional expected RV

\footnotetext{
${ }^{4}$ In an earlier version of the paper, the second term on the right-hand-side of equation (2.7) was excluded. This sometimes generates a better fit empirically, but a positive conditional variance cannot be ensured in this case.
} 
as

$$
\begin{aligned}
h_{t+1}^{R V} & =\omega_{2}+\left(\theta+\beta_{2}\right) h_{t}^{R V}+\kappa \alpha_{2} \beta_{2} \gamma_{2}^{2} h_{t}^{R}+\beta_{2} \alpha_{2}\left[\left(\varepsilon_{2, t+1}-\gamma_{2} \sqrt{\bar{h}_{t}}\right)^{2}-\left(1+\gamma_{2}^{2} \bar{h}_{t}\right)\right] \\
& =\omega_{2}-\beta_{2} \alpha_{2}+\left(\theta+\beta_{2}-(1-\kappa) \alpha_{2} \beta_{2} \gamma_{2}^{2}\right) h_{t}^{R V}+\beta_{2} \alpha_{2}\left(\varepsilon_{2, t+1}-\gamma_{2} \sqrt{\bar{h}_{t}}\right)^{2}
\end{aligned}
$$

which shows that it has a structure very similar to the GARCH dynamic in (2.6). Note also from (2.11) that the following sufficient conditions ensure the positivity of $h_{t+1}^{R V}$

$$
\begin{aligned}
& \tilde{\alpha}_{2}=\beta_{2} \alpha_{2} \geq 0 \\
& \tilde{\omega}_{2}=\omega_{2}-\tilde{\alpha}_{2} \geq 0 \\
& \tilde{\beta}_{2}=\theta+\beta_{2}-(1-\kappa) \tilde{\alpha}_{2} \gamma_{2}^{2} \geq 0
\end{aligned}
$$

The new model is completed by defining the total conditional variance, $\bar{h}_{t}$, as a weighted average of the two variance components

$$
\bar{h}_{t}=\kappa h_{t}^{R}+(1-\kappa) h_{t}^{R V}
$$

where the weight $\kappa$ is a parameter to be estimated.

In sum the new model is defined by

$$
\begin{aligned}
R_{t+1} & =r+\lambda \bar{h}_{t}-\frac{1}{2} \bar{h}_{t}+\sqrt{\bar{h}_{t}} \varepsilon_{1, t+1}, \text { with } \\
\bar{h}_{t} & =\kappa h_{t}^{R}+(1-\kappa) h_{t}^{R V}, \text { where } \\
h_{t+1}^{R} & =\omega_{1}+\beta_{1} h_{t}^{R}+\alpha_{1}\left(\varepsilon_{1, t+1}-\gamma_{1} \sqrt{\bar{h}_{t}}\right)^{2}, \\
R V_{t+1} & =h_{t}^{R V}+\alpha_{2}\left[\left(\varepsilon_{2, t+1}-\gamma_{2} \sqrt{\bar{h}_{t}}\right)^{2}-\left(1+\gamma_{2}^{2} \bar{h}_{t}\right)\right], \text { and } \\
h_{t+1}^{R V} & =\tilde{\omega}_{2}+\tilde{\beta}_{2} h_{t}^{R V}+\tilde{\alpha}_{2}\left(\varepsilon_{2, t+1}-\gamma_{2} \sqrt{\bar{h}_{t}}\right)^{2}
\end{aligned}
$$

and we will refer to it as the Generalized Affine RV model or GARV for short. We allow for dependence between the $\varepsilon_{1, t+1}$ and $\varepsilon_{2, t+1}$ by assuming they follow a bivariate standard normal distribution with correlation $\rho$.

Note that the GARCH model in Section 2.1 appears as a special case of GARV when $\kappa=1$. A model purely based on expected realized volatility emerges if $\kappa=0$. We denote this special case by ARV and discuss it further below.

The following dynamic model-implied moments will be key for understanding the models' per- 
formance in fitting returns and options. First note the expected variance one day ahead is

$$
E_{t}\left(\bar{h}_{t+1}\right)=\kappa E_{t}\left(h_{t+1}^{R}\right)+(1-\kappa) E_{t}\left(h_{t+1}^{R V}\right),
$$

where

$$
\begin{aligned}
& E_{t}\left(h_{t+1}^{R}\right)=\omega_{1}+\alpha_{1}+\left(\beta_{1}+\alpha_{1} \gamma_{1}^{2} \kappa\right) h_{t}^{R}+\alpha_{1} \gamma_{1}^{2}(1-\kappa) h_{t}^{R V} \\
& E_{t}\left(h_{t+1}^{R V}\right)=\tilde{\omega}_{2}+\tilde{\alpha}_{2}+\tilde{\alpha}_{2} \gamma_{2}^{2} \kappa h_{t}^{R}+\left(\tilde{\beta}_{2}+\tilde{\alpha}_{2} \gamma_{2}^{2}(1-\kappa)\right) h_{t}^{R V}
\end{aligned}
$$

Having two components in the variance allows for richer variance dynamics and richer term structures of variance, which is crucial for valuing options with maturities of several months.

Second, the conditional variance of variance implied by the model can be derived as

$$
\operatorname{Var}_{t}\left(\bar{h}_{t+1}\right)=2 \kappa^{2} \alpha_{1}^{2}\left(1+2 \gamma_{1}^{2} \bar{h}_{t}\right)+2(1-\kappa)^{2} \alpha_{2}^{2} \beta_{2}^{2}\left(1+2 \gamma_{2}^{2} \bar{h}_{t}\right)+4 \rho \alpha_{1} \alpha_{2} \beta_{2} \kappa(1-\kappa)\left(\rho+2 \gamma_{1} \gamma_{2} \bar{h}_{t}\right) .
$$

Third, the conditional covariance between return and variance is

$$
\operatorname{Cov}_{t}\left(R_{t+1}, \bar{h}_{t+1}\right)=-2\left(\kappa \alpha_{1} \gamma_{1}+(1-\kappa) \beta_{2} \alpha_{2} \gamma_{2} \rho\right) \bar{h}_{t}
$$

Notice that using RV as a factor in the variance dynamic not only provides potentially more accurate modeling of the current spot volatility and the volatility term structure, it also provides more flexible functional forms for variance of variance and the leverage effect, which is crucial in option valuation. We plot the time series paths of these moments in our empirical work below.

Below we will be estimating the model using standard quasi maximum likelihood techniques (QMLE). We have observations on $R_{t}$ and $R V_{t}$, and for estimation we need to derive their moments as implied by the model. We already have the expected value of returns and of RV from (2.5) and (2.7). In addition we need the conditional variance of RV

$$
\operatorname{Var}_{t}\left(R V_{t+1}\right)=\alpha_{2}^{2}\left(2+4 \gamma_{2}^{2} \bar{h}_{t}\right)
$$

and the conditional covariance between RV and returns

$$
\operatorname{Cov}_{t}\left(R V_{t+1}, R_{t+1}\right)=-2 \rho \gamma_{2} \alpha_{2} \bar{h}_{t}
$$

The GARV model has twelve parameters in total, and it may prove useful to also investigate a more parsimonious special case where only the RV component plays a role in the variance dynamic. We now define such a model which we denote ARV. 


\subsection{The Affine RV (ARV) Model as a Special Case}

As noted above, we will refer to the special case of the GARV model with $\kappa=0$ as the ARV model. In this case $\bar{h}_{t}=h_{t}^{R V}$ and so daily returns are defined by

$$
R_{t+1}=r+\lambda h_{t}^{R V}-\frac{1}{2} h_{t}^{R V}+\sqrt{h_{t}^{R V}} \varepsilon_{1, t+1} .
$$

The conditional variance is now simply

$$
h_{t}^{R V}=\omega_{2}+\theta h_{t-1}^{R V}+\beta_{2} R V_{t}
$$

and the observed RV is again modeled as

$$
R V_{t+1}=h_{t}^{R V}+\alpha_{2}\left[\left(\varepsilon_{2, t+1}-\gamma_{2} \sqrt{h_{t}^{R V}}\right)^{2}-\left(1+\gamma_{2}^{2} h_{t}^{R V}\right)\right] .
$$

By substituting (2.16) into (2.15) we get

$$
\begin{aligned}
h_{t+1}^{R V} & =\omega_{2}+\left(\theta+\beta_{2}\right) h_{t}^{R V}+\beta_{2} \alpha_{2}\left[\left(\varepsilon_{2, t+1}-\gamma_{2} \sqrt{h_{t}^{R V}}\right)^{2}-\left(1+\gamma_{2}^{2} h_{t}^{R V}\right)\right] \\
& =\left(\omega_{2}-\beta_{2} \alpha_{2}\right)+\left(\theta+\beta_{2}-\beta_{2} \alpha_{2} \gamma_{2}^{2}\right) h_{t}^{R V}+\beta_{2} \alpha_{2}\left(\varepsilon_{2, t+1}-\gamma_{2} \sqrt{h_{t}^{R V}}\right)^{2} \\
& \equiv \tilde{\omega}_{2}+\tilde{\beta}_{2} h_{t}^{R V}+\tilde{\alpha}_{2}\left(\varepsilon_{2, t+1}-\gamma_{2} \sqrt{h_{t}^{R V}}\right)^{2}
\end{aligned}
$$

which shows that the ARV dynamic is exactly of the GARCH form in (2.1).

As a practical matter, estimating first the standard GARCH model and the simple ARV model can provide good starting values for the parameters in the general GARV model.

The key dynamic moments implied by the ARV model are as follows. First, the expected variance is given by

$$
E_{t}\left(h_{t+1}^{R V}\right)=\omega_{2}+\left(\theta+\beta_{2}\right) h_{t}^{R V} .
$$

Second, the conditional variance of variance implied is

$$
\operatorname{Var}_{t}\left(h_{t+1}^{R V}\right)=2 \beta_{2}^{2} \alpha_{2}^{2}\left(1+2 \gamma_{2}^{2} h_{t}^{R V}\right) .
$$


Third, the conditional covariance between return and variance is

$$
\operatorname{Cov}_{t}\left(R_{t+1}, h_{t+1}^{R V}\right)=-2 \beta_{2} \alpha_{2} \gamma_{2} \rho h_{t}^{R V} .
$$

The conditional moments needed for QMLE estimation of the ARV model are

$$
\operatorname{Cov}_{t}\left(R_{t+1}, R V_{t+1}\right)=-2 \alpha_{2} \gamma_{2} \rho h_{t}^{R V}
$$

and

$$
\operatorname{Var}_{t}\left(R V_{t+1}\right)=2 \alpha_{2}^{2}\left(1+2 \gamma_{2}^{2} h_{t}^{R V}\right) .
$$

Armed with the model specifications and moments for the GARCH, GARV and ARV models, we are now ready to embark on their empirical estimation.

\section{Daily Return and Realized Volatility Empirics}

We now estimate the three models described in Section 2, namely, GARCH, GARV, and ARV. The GARCH model can be estimated using only daily returns, whereas the GARV and ARV models require RV data as well.

We use daily close-to-close S\&P 500 futures returns and realized variance data computed from intraday S\&P 500 futures prices for the period January 2, 1990 to December 30, 2010, which yields a total of 5,243 daily observations.

To construct the daily RV series, we start from a one-minute grid of prices constructed from open to close each day. One-minute returns are likely to be contaminated by market microstructure effects and so we compute RV as the sum of 5-minute squared returns starting each day from the first price on the one-minute grid. We then compute a second RV estimate starting from the second price on the one-minute grid using again the sum of squared five-minute returns. We continue until we have five RV estimates for each day based on five different subsets of prices on the one-minute grid. As suggested in Zhang, Mykland, and Aït-Sahalia (2005), we compute the Average RV time series as the simple average of the five available RV estimates on each day. We use a multiplicative scaling of the Average RV series to match the unconditional variance of S\&P 500 returns. ${ }^{5}$

The daily returns on the S\&P500 index futures are plotted in the top panel of Figure 1. The dramatic daily returns observed during the 2008-2009 equity market crash dominate the picture. The low-volatility periods in the mid 1990s and mid 2000s are also evident, as are the periods of high volatility in the early 1990s, and in the late 1990s and early 2000s. The bottom panel of Figure

\footnotetext{
${ }^{5}$ Hansen and Lunde (2005) discusses various approaches to adjusting open-to-close RVs.
} 
1 plots the daily realized volatility (square root of RV) using the Average RV estimates.

In order to assess the dynamic properties of the return and RV series we plot their autocorrelation functions (ACFs) for lag 1 through 60 in the top panels of Figure 2. The horizontal lines denote a Bartlett two-standard error confidence around zero. As is typically found, returns have very little persistence, whereas RV is very highly persistent. The second row of panels in Figure 2 show the ACFs for square returns and squared RVs. The squared returns are persistent, capturing volatility clustering albeit more crudely than the RVs. The square RVs are also quite persistent suggesting evidence of variance of variance dynamics. The bottom panel plots the ACF of the cross product of returns and RV which may capture dynamics in the leverage effect. While quite a few of the autocorrelations are significant, there is not a clear cut dynamic pattern apparent.

The Average RVs in Figure 1 incorporate a lot of intraday information and thus are much smoother than for example squared daily returns from closing prices. But it is still the case that further smoothing of RV in the dynamic RV models may be required. Our GARV and ARV models accommodate this by modeling the dynamics of conditionally expected RV, which we denote above by $h_{t}^{R V}$, rather than the raw RV itself.

\subsection{Maximum Likelihood Estimation on Returns and RV}

We estimate the three models using quasi maximum likelihood. ${ }^{6}$ The quasi-log-likelihood of returns at time $t+1$ conditional on information known at time $t$ is defined using the normal distribution

$$
L\left(R_{t+1} \mid I_{t}\right)=-\frac{1}{2} \ln \left(2 \pi \operatorname{Var}_{t}\left[R_{t+1}\right]\right)-\frac{\left(R_{t+1}-E_{t}\left[R_{t+1}\right]\right)^{2}}{2 \operatorname{Var}_{t}\left[R_{t+1}\right]}
$$

The quasi-log-likelihood of realized variance at time $t+1$ conditional on information known at time $t$ is similarly

$$
L\left(R V_{t+1} \mid I_{t}\right)=-\frac{1}{2} \ln \left(2 \pi \operatorname{Var}_{t}\left[R V_{t+1}\right]\right)-\frac{\left(R V_{t+1}-E_{t}\left[R V_{t+1}\right]\right)^{2}}{2 \operatorname{Var}_{t}\left[R V_{t+1}\right]} .
$$

and the joint quasi-log-likelihood of returns and realized variance is defined using the log of the bivariate normal distribution, call it $L\left(R_{t+1}, R V_{t+1} \mid I_{t}\right)$, which depends on the first two conditional moments of $\left\{R_{t+1}, R V_{t+1}\right\}$. These conditional moments are provided in Section 2 above.

Summing the log likelihoods over all the observations generates the sample return likelihood function

$$
\ln L^{R}=\sum_{t=1}^{T-1} L\left(R_{t+1}, R V_{t+1} \mid I_{t}\right)
$$

\footnotetext{
${ }^{6}$ Gourieroux, Monfort and Trognon (1984) and Bollerslev and Wooldridge (1992) develop sufficient conditions for consistency of QMLE.
} 
The QMLE estimation results are reported in Table 1. Note that we estimate the unconditional variance, $E\left[\bar{h}_{t}\right]$ as a parameter and imply the $\omega$ parameters from the unconditional variance formula in Appendix A. ${ }^{7}$ Appendix A also contains details on persistence and stationarity conditions.

For the GARCH and ARV models, the weighting parameter $\kappa$ is set equal to one and zero, respectively. For the GARV model, Table 1 indicates that the point estimate of the parameter $\kappa$ is 0.395, which is more than two standard deviations away from either 0 (ARV) or 1 (GARCH).

The persistence of volatility and the volatility components is indicated at the bottom of Table 1. The persistence of the GARCH variance is 0.9661, which is in line with existing results in the literature. The persistence for the ARV model is 0.9796. In the GARV model, the persistence associated with the GARCH component is rapidly mean-reverting at 0.3796, whereas the persistence associated with the realized volatility component is $0.5922 .{ }^{8}$

The estimates of $\gamma_{1}$ and $\gamma_{2}$ are positive in all cases, which confirms the commonly found leverage effect and which suggests negative skewness of the return distribution. The estimate of $\rho$ is approximately 0.1 in both ARV and GARV models. Note that the sign of $\rho$ does not in itself determine leverage, as can be seen from (2.13), and the positive estimate of $\rho$ is consistent with negative skewness.

The log-likelihood values allow us to test the special cases of the ARV model against the more general GARV model. Using the standard asymptotic distribution, the restrictions imposed by the ARV model are resoundingly rejected, indicating that the GARCH and RV dynamic in the GARV model both contribute to the modeling of daily index returns. ${ }^{9}$

It is less straightforward to statistically compare the GARCH model and the two newly proposed models. The GARCH likelihood does not contain a realized volatility component, and so cannot be meaningfully compared to the overall likelihood of the ARV and GARV models. We therefore perform an additional estimation of ARV and GARV optimizing the likelihood on returns only. This is done by computing the conditional mean and variance of returns implied by the model, and then maximizing the univariate Gaussian likelihood using these two conditional moments. These returnbased log-likelihood results are reported in the row of Table 1 labeled "Maximized on Returns". They show that the Heston-Nandi GARCH model is dominated by the ARV and GARV models.

\footnotetext{
${ }^{7}$ Estimating $E\left[\bar{h}_{t}\right]$ implies $\omega_{1}$ in the GARCH model and $\tilde{\omega}_{2}$ in the ARV model. In the GARV model we further have that $E\left[h_{t}^{R V}\right]=E\left[h_{t}^{R}\right]$ so that both $\omega_{1}$ and $\tilde{\omega}_{2}$ are implied in this model.

${ }^{8}$ For the GARV model we report variance persistence using the diagonal elements in the $\phi_{1}$ matrix in Appendix A. This facilitates comparison with the pure GARCH and ARV models. Alternatively, we could report the eigenvalues of the $\phi_{1}$ matrix.

${ }^{9}$ Note that standard asymptotics may not apply here because certain parameters are not identified under the null hypothesis (Andrews, 1993; Andrews and Ploberger, 1994). Similar problems arise for instance when one tests GARCH models (Andrews, 2001) or regime switching models (Cho and White, 2007). However, the difference between our likelihoods is so large that the inference from standard asymptotics is unlikely to be overturned.
} 
Figure 3 plots the ACFs of the GARV model residuals, $\varepsilon_{1, t}$ and $\varepsilon_{2, t}$ and their squares and cross products. Comparing Figures 2 and 3, it appears that the GARV model has adequately captured the persistent dynamics in the RV, squared returns, and squared RVs. The ACFs of the ARV model residuals look quite similar to those reported for GARV in Figure 3 but we do not report them here in order to save space. Our QMLE estimator requires that the dynamics in the first and second moments of returns and RV are adequately specified, and Figure 3 provides an important diagnostic in this regard.

Overall, the estimation based on returns and realized volatility yields two important conclusions. First, both the GARCH volatility and the realized volatility dynamic contribute to the modeling of daily index volatility in our framework. Second, the new ARV and GARV models, which incorporate RV information, both offer a better description of the distribution of index returns than does the basic GARCH model.

\subsection{Dynamic Model Properties}

Figures 4-6 report on various dynamic properties of the three models we have estimated on returns using QMLE in Table 1.

Figure 4 plots the daily conditional volatility $\sqrt{\bar{h}_{t}}=\sqrt{\operatorname{Var}_{t}\left(R_{t+1}\right)}$ for each of the three models. Not surprisingly, all models track the market volatility during the 1990-2010 period in a similar way. Notice however, that the ARV model in the middle panel tends to display stronger spikes in volatility than does the GARCH model in the top panel. The GARV model in the bottom panel appears to fall in between GARCH and ARV in this regard. This is sensible as it can be viewed as a weighted average of the GARCH and ARV models.

Figure 5 confirms the impression from Figure 4 by plotting the model-implied conditional volatility of variance, defined as $\sqrt{\operatorname{Var}_{t}\left(\bar{h}_{t+1}\right)}$. The volatility of variance is generally higher in the ARV model than in the other models, and it also tends to show more high-frequency moments inherited from $\bar{h}_{t}$. The volatility of variance in the GARV model in the bottom panel again falls between ARV and GARCH in the top panel.

Figure 6 plots the model-implied conditional correlation between return and variance, defined as

$$
\operatorname{Corr}_{t}\left(R_{t+1}, \bar{h}_{t+1}\right)=\frac{\operatorname{Cov}_{t}\left(R_{t+1}, \bar{h}_{t+1}\right)}{\sqrt{\operatorname{Var}_{t}\left(\bar{h}_{t+1}\right) \bar{h}_{t}}} .
$$

Figure 6 shows that the models differ considerably in this regard. The conditional correlation fluctuates around -0.9 in the GARCH model, is constant at around -0.1 in the ARV model, and fluctuates around -0.7 in the GARV model. 
Recall that the GARV model has two sources of the leverage effect: $\gamma_{1}$ from the GARCH part and $\rho \gamma_{2}$ from the RV part. Clearly, allowing for the GARCH to play a role in the models increases the estimated leverage effect. This is important to keep in mind when analyzing the models' ability to fit options, to which we turn next.

\section{Risk Neutralization and Option Valuation}

In this section we use the return processes defined above to derive option valuation formulas, using the models' conditional moment generating functions. We present results for the GARV model only, as the ARV model can be obtained as a special case. Option valuation in the GARCH model can be done using the results in Heston and Nandi (2000).

\subsection{The Moment-Generating Function}

The affine structure of the GARV model ensures that the moment generating function (MGF) exists and is exponentially affine. Appendix B shows that the one-period joint conditional moment generating function for $R_{t+1}, h_{t+1}^{R}$ and $h_{t+1}^{R V}$ is of the form

$$
\begin{aligned}
& E_{t}\left[\exp \left(u R_{t+1}+w_{R} h_{t+1}^{R}+w_{R V} h_{t+1}^{R V}\right)\right] \\
= & \exp \left(A_{1}\left(u, w_{R}, w_{R V}\right) h_{t}^{R}+A_{2}\left(u, w_{R}, w_{R V}\right) h_{t}^{R V}+B\left(u, w_{R}, w_{R V}\right)\right),
\end{aligned}
$$

In Appendix B we use this one-period joint MGF to derive the MGF for the multiperiod aggregate return

$$
\Psi_{t, t+M}(u) \equiv E_{t}\left[\exp \left(u \sum_{j=1}^{M} R_{t+j}\right)\right]=\exp \left(C_{1}(u, M) h_{t}^{R}+C_{2}(u, M) h_{t}^{R V}+D(u, M)\right)
$$

\subsection{Risk Neutralization}

We follow the risk-neutralization approach of Christoffersen, Elkamhi, Feunou and Jacobs (2010). Appendix B contains the details. The GARV model has two shocks, and so we rely on the following pricing kernel

$$
Z_{t+1}=\frac{\exp \left(\nu_{1, t} \varepsilon_{1, t+1}+\nu_{2, t} \varepsilon_{2, t+1}\right)}{E_{t}\left[\exp \left(\nu_{1, t} \varepsilon_{1, t+1}+\nu_{2, t} \varepsilon_{2, t+1}\right)\right]}
$$

From this pricing kernel and by imposing that the risk-neutral dynamic is of the same form as 
the physical, we can derive the following risk-neutral process

$$
\begin{aligned}
R_{t+1} & =r-\frac{1}{2} \bar{h}_{t}+\sqrt{\bar{h}_{t}} \varepsilon_{1, t+1}^{*} \\
\bar{h}_{t} & =\kappa h_{t}^{R}+(1-\kappa) h_{t}^{R V}
\end{aligned}
$$

with

$$
\begin{aligned}
h_{t+1}^{R} & =\omega_{1}+\beta_{1} h_{t}^{R}+\alpha_{1}\left(\varepsilon_{1, t+1}^{*}-\gamma_{1}^{*} \sqrt{\bar{h}_{t}}\right)^{2} \\
R V_{t+1} & =h_{t}^{R V *}+\alpha_{2}\left[\left(\varepsilon_{2, t+1}^{*}-\gamma_{2}^{*} \sqrt{\bar{h}_{t}}\right)^{2}-\left(1+\gamma_{2}^{* 2} \bar{h}_{t}\right)\right] \\
h_{t+1}^{R V} & =\tilde{\omega}_{2}+\tilde{\beta}_{2} h_{t}^{R V}+\tilde{\alpha}_{2}\left(\varepsilon_{2, t+1}^{*}-\gamma_{2}^{*} \sqrt{\bar{h}_{t}}\right)^{2} \\
h_{t}^{R V *} & =h_{t}^{R V}+\alpha_{2}\left(\gamma_{2}^{* 2}-\gamma_{2}^{2}\right) \bar{h}_{t},
\end{aligned}
$$

where $\varepsilon_{1, t+1}^{*}$ and $\varepsilon_{2, t+1}^{*}$, are bivariate standard normal with correlation $\rho$ under $Q$.

The mapping from physical to risk neutral innovations is

$$
\begin{aligned}
& \varepsilon_{1, t+1}^{*}=\varepsilon_{1, t+1}-\left(\nu_{1, t}+\nu_{2, t} \rho\right)=\varepsilon_{1, t+1}+\lambda \sqrt{\bar{h}_{t}} \\
& \varepsilon_{2, t+1}^{*}=\varepsilon_{2, t+1}-\left(\nu_{2, t}+\nu_{1, t} \rho\right)=\varepsilon_{2, t+1}-\chi \sqrt{\bar{h}_{t}}
\end{aligned}
$$

and the mapping from physical to risk-neutral parameters is

$$
\begin{aligned}
& \gamma_{1}^{*}=\gamma_{1}+\lambda \\
& \gamma_{2}^{*}=\gamma_{2}-\chi
\end{aligned}
$$

where $\chi$ is a risk price compensation for the innovations to RV. Under the risk-neutral measure we of course have that the price of equity risk is zero.

The risk-neutral MGF is easily obtained using the physical MGF in (4.1) and the parameter mapping in (4.3). We can write

$$
\Psi_{t, t+M}^{Q}(u)=C_{1}^{*}(u, M) h_{t}^{R}+C_{2}^{*}(u, M) h_{t}^{R V}+D^{*}(u, M) .
$$

\subsection{Option Valuation}

Using these results, the price at time $t$ of a European call option with payoff $\left(S_{t+M}-X\right)^{+}$at time $t+M$ is given by

$$
C(t, M)=S_{t} P_{1}(t, M)-\exp (-r M) X P_{2}(t, M) .
$$


The risk neutral probabilities $P_{1}(t, M)$ and $P_{2}(t, M)$ can be computed using Fourier inversion of the risk-neutral conditional characteristic function as follows

$$
\begin{aligned}
& P_{1}(t, M)=\frac{1}{2}+\int_{0}^{+\infty} \operatorname{Re}\left[\frac{\exp \left(\Psi_{t, t+M}^{Q}(1+i u)-r M-i u \ln \left(\frac{X}{S_{t}}\right)\right)}{\pi i u}\right] d u \\
& P_{2}(t, M)=\frac{1}{2}+\int_{0}^{+\infty} \operatorname{Re}\left[\frac{\exp \left(-i u \ln \left(\frac{X}{S_{t}}\right)+\Psi_{t, t+M}^{Q}(i u)\right)}{\pi i u}\right] d u .
\end{aligned}
$$

where the risk-neutral characteristic function is available using the risk-neutral MGF in (4.4). Put options can be valued using put-call parity.

\section{Option-Based Estimation}

We now discuss the option fit of the two new models, and compare it with the fit of the benchmark GARCH model. We first discuss the option data used in our empirical analysis. Then we estimate the models by maximizing the fit on our option data. Throughout, we use returns and RV to filter volatility.

\subsection{Option Data}

We use closing prices on European S\&P500 index options from OptionMetrics for the period January 10, 1996 through October 28, 2009. In order to ensure that the contracts we use are liquid, we rely only on out-of-the-money options with maturity between 15 and 180 days. For each maturity on each Wednesday, we retain only the six most liquid strike prices. We restrict attention to Wednesday data. This enables us to study a fairly long time-period while keeping the size of the data set manageable. Our sample contains 14,276 options. In order to facilitate computation and interpretation we use put-call parity to convert the out-of-the-money put options to in-the-money call options.

Table 2 describes key features of the data. The top panel of Table 2 sorts the data by six moneyness categories and reports the number of contracts, the average option price, the average Black-Scholes implied volatility, and the average bid-ask spread in dollars. Moneyness is measured using the Black-Scholes delta defined as

$$
\text { Delta }=N\left(\frac{\ln \left(S_{t} / X\right)+r M-1 / 2\left(I V^{M k t}\right)^{2} M / 365}{I V^{M k t} \sqrt{M / 365}}\right)
$$


where $N(*)$ denotes the normal CDF and $I V^{M k t}$ denotes the annualized implied Black-Scholes volatility computed at the market price of the option. The row reporting average implied volatility shows that deep out-of-the-money puts, those with deltas higher than 0.7 , are relatively expensive. The implied volatility for those options is $24.52 \%$, compared with $19.25 \%$ for options with delta between 0.4 and 0.5 , and $17.26 \%$ for options with delta below 0.3 . The data thus display the well-known smirk pattern across moneyness.

The middle panel sorts the data by maturity reported in calendar days. The implied volatility row shows that the term structure of volatility is roughly flat on average during the sample period.

The bottom panel in Table 2 sorts the data by the VIX volatility level. Obviously option prices and $I V$ s are increasing in VIX, and dollar spreads are increasing in VIX as well. More importantly, note that most of our data are from days with VIX levels between 15 and $35 \%$.

\subsection{Fitting Options while Filtering Volatility on $\mathbf{R}$ and RV}

As is standard in the derivatives literature, we next compare the GARCH, ARV and GARV models using the implied volatility root mean squared error (IVRMSE). We refer to Renault (1997) for a discussion on the benefits of using the IVRMSE metric for comparing option pricing models. For the computation of the IVRMSE, we invert each computed model option price $C_{j}^{\text {Mod }}$ using the Black-Scholes formula, $B S M$, to get the implied volatilities $I V_{j}^{M o d}$

$$
I V_{j}^{M o d}=B S M^{-1}\left(C_{j}^{M o d}\right)
$$

We compare these model IVs to the market IV from the option data set, denoted $I V_{j}^{M k t}$, which are also computed by inverting Black-Scholes

$$
I V_{j}^{M k t}=B S M^{-1}\left(C_{j}^{M k t}\right)
$$

With $N$ denoting the total number of options in the sample, the IVRMSE is now computed as

$$
I V R M S E \equiv \sqrt{\frac{1}{N} \sum_{j=1}^{N}\left(I V_{j}^{M k t}-I V_{j}^{M o d}\right)^{2}}
$$

Estimating model parameters by minimizing IVRMSE is numerically intensive, because the $B S M$ inversion must be done for each set of model option prices tried by the optimizer. Instead of minimizing IVRMSE, we therefore rely on Trolle and Schwartz (2009), who minimize vega-weighted 
RMSE (VWRMSE)

$$
V W R M S E \equiv \sqrt{\frac{1}{N} \sum_{j=1}^{N} e_{j}^{2}} \equiv \sqrt{\frac{1}{N} \sum_{j=1}^{N}\left(\left(C_{j}^{M k t}-C_{j}^{M o d}\right) / B S V_{j}^{M k t}\right)^{2}},
$$

where $B S V_{j}^{M k t}$ represents the Black-Scholes vega of the option (the derivative with respect to volatility) computed using the market implied level of volatility. IVRMSE and VWRMSE are generally similar in value. In the tables below we will report both IVRMSE and VWRMSE, but we will focus our discussion on the IVRMSEs.

Instead of minimizing VWRMSE directly, we estimate the risk neutral parameters by maximizing the Gaussian vega-weighted option error likelihood ${ }^{10}$

$$
\ln L^{O} \propto-\frac{1}{2} \sum_{j=1}^{N}\left\{\ln \left(V W R M S E^{2}\right)+e_{j}^{2} / V W R M S E^{2}\right\} .
$$

Table 3 contains the results of the option-based estimation. Note that because we are estimating the model on options only, we obtain risk-neutral parameters, and we do not identify the two prices of risk, $\lambda$ and $\chi$. Note also that while we are fitting option IVs, we continue to filter volatility on returns and RV so as to force model consistency in the estimation. We again estimate $E^{Q}[\bar{h}]$ and imply the $\omega$ estimates from the unconditional risk neutral variance expression.

At the bottom of Table 3 we report the IVRMSE metric. The IVRMSE for the GARV model is 3.145 , compared with 4.603 for the benchmark GARCH model. This is an improvement of $32.7 \%$, which is very impressive. The IVRMSE of the ARV model is 3.467, which is an improvement of $24.7 \%$ over GARCH. The GARV model outperforms the ARV model by about $9.3 \%$, which indicates that the GARCH-type volatility dynamic does contribute somewhat to option valuation in addition to the RV based dynamic, but clearly the RV dynamics alone yields a clear improvement over the pure GARCH model.

The variance components are more persistent in Table 3 than in Table 1 . This is a common finding in the derivatives literature: Risk neutral variance is more persistent than physical variance. Note that in the case of the GARV model, both components are now very persistent.

Comparing the option-based parameter estimates in Table 3 with the estimates in Table 1 based on returns and realized volatility, the estimate of $\kappa$ in the GARV model is substantially lower in Table 3, suggesting that for our model specifications the economic value of including RV in option pricing models is high.

\footnotetext{
${ }^{10}$ Below we will estimate the models maximizing the joint likelihood on returns and options. For comparison, we therefore maximize the option likelihood here instead of minimizing VWRMSE.
} 
All estimates of $\gamma_{1}^{*}$ and $\gamma_{2}^{*}$ are positive in Table 3, and for the GARV model they are larger than in Table 1. Estimates of $\rho$ are higher in Table 3 compared with Table 1 and $\rho$ hits the boundary of 1 for the GARV model, suggesting that the risk-neutral skewness is larger (in magnitude) than its physical counterpart. Similar results are commonly found in the literature.

Overall, the results from model estimation based on option data confirm the main conclusions from QMLE estimation on realized volatility and returns in Section 3. First, realized volatility contains important information that is not contained in lagged squared returns. Second, GARCH volatility dynamics do offer some additional contribution to the option fit in RV-based models.

\subsection{Decomposing Model Fit}

We now dissect the overall IVRMSE results reported in Table 3 by sorting the data by moneyness, maturity and VIX levels, using the bins from Table 2. Table 4 contains the decomposed IVRMSE results.

Consider first Panel A of Table 4 which reports the IVRMSE for the three models by moneyness bins corresponding to those used in Table 2. Looking across columns, we see that the GARV model, which had the lowest overall IVRMSE in Table 4, has the lowest IVRMSE in each of the six moneyness categories considered. The benefits offered by the GARV model are therefore not restricted to any particular subset of strike prices. The performance of the ARV model is also consistent across strikes. Notice also that all models tend to perform worst for deep out-of-themoney put options $($ Delta $>0.7)$, which also have the highest average implied volatility (see Table $2)$.

Consider now Panel B in Table 4 which reports the IVRMSE across maturity categories. Again we see that the GARV model performs the best in all six maturity categories and the ARV is second best everywhere. All models have relatively more difficulty fitting the very short maturity and the longest-maturity options.

Panel C reports the IVRMSE across VIX levels. The GARV model is now best in five of the six categories. When the VIX is between 15 and $20 \%$ then the ARV model is slightly better. Not surprisingly, all models have most difficulty fitting options when the level of market volatility is high.

Figure 7 gives a visual impression of the decomposition of IVRMSEs in the different models. The impressive performance of the two RV-based models across moneyness (top panel), maturity (middle panel) and VIX level (bottom level) is readily apparent.

In Figure 8, we complement the results in Tables 3-4 and Figure 7 by plotting the weekly IVRMSE over time. We use only at-the-money (ATM) options for this Figure. The top panel in 
Figure 8 shows the ARV (solid line) and GARCH (dashes), and the bottom panel show the GARV (solid line) and GARCH (dashes).

The weekly IVRMSE clearly contain much high-frequency variation. Nevertheless, Figure 8 shows that during most of the sample, the IVRMSE in the RV based models outperform the IVRMSE in the GARCH model.

\section{Joint Estimation on Returns, RV, and Options}

Estimating model parameters from option data is insightful, but the resulting parameters are uninformative about certain model properties. Most critically, they remain silent about risk premia, and therefore about the assumptions regarding the pricing kernel. Bates (1996) observed that the most critical shortcoming of existing option pricing models is their inability to jointly fit returns and options, and this shortcoming has not yet been fully addressed in the literature. We therefore also estimate the newly proposed models using data on returns, RV, and options jointly. We maximize likelihoods that are a combination of the joint quasi-log-likelihood of returns and realized variance $\ln L^{R}$ in (3.2) and an options component. As above, we use the Black-Scholes Vega (BSV) weighted option valuation errors defined as

$$
e_{j}=\left(C_{j}^{M k t}-C_{j}^{M o d}\right) / B S V_{j}^{M k t},
$$

and apply again the Gaussian log likelihood

$$
\ln L^{O} \propto-\frac{1}{2} \sum_{j=1}^{N}\left\{\ln \left(V W R M S E^{2}\right)+e_{j}^{2} / V W R M S E^{2}\right\}
$$

Using the two likelihoods in (3.2) and (6.1), we are ready to solve the following joint optimization problem

$$
\max \ln L^{R}+\ln L^{O},
$$

with respect to the physical parameters and the risk premia mapping the physical to the risk-neutral parameters.

We pay particular attention to the risk-neutralization and the resulting risk premia. The results in Appendix A imply that for the GARV model, the premia associated with the GARCH and RV 
components are, respectively

$$
\begin{aligned}
& E_{t}^{Q}\left[h_{t+1}^{R}\right]-E_{t}\left[h_{t+1}^{R}\right]=\alpha_{1}\left(\gamma_{1}^{* 2}-\gamma_{1}^{2}\right) \bar{h}_{t}, \text { and } \\
& E_{t}^{Q}\left[h_{t+1}^{R V}\right]-E_{t}\left[h_{t+1}^{R V}\right]=\tilde{\alpha}_{2}\left(\gamma_{2}^{* 2}-\gamma_{2}^{2}\right) \bar{h}_{t} .
\end{aligned}
$$

It is well-known in the literature that option valuation models must be able to generate risk-neutral variances that are larger than physical variances in order to be empirically relevant. Now recall from above that

$$
\begin{aligned}
& \gamma_{1}^{*}=\gamma_{1}+\lambda \\
& \gamma_{2}^{*}=\gamma_{2}-\chi
\end{aligned}
$$

so that $E_{t}^{Q}\left[h_{t+1}^{R}\right]-E_{t}\left[h_{t+1}^{R}\right]>0$ requires $\lambda>0$ because $\alpha_{1}>0$ and $\bar{h}_{t}>0$. Similarly $E_{t}^{Q}\left[h_{t+1}^{R V}\right]-$ $E_{t}\left[h_{t+1}^{R V}\right]>0$ requires $\chi<0$ because $\tilde{\alpha}_{2}>0$ and $\bar{h}_{t}>0$.

In the special case of the ARV model the variance risk premium has only one component, namely

$$
E_{t}^{Q}\left[h_{t+1}^{R V}\right]-E_{t}\left[h_{t+1}^{R V}\right]=\tilde{\alpha}_{2}\left(\gamma_{2}^{* 2}-\gamma_{2}^{2}\right) h_{t}^{R V}
$$

which is ultimately driven by $\chi$. In the special case of the GARCH model the variance risk premium also has only one component, namely

$$
E_{t}^{Q}\left[h_{t+1}^{R}\right]-E_{t}\left[h_{t+1}^{R}\right]=\alpha_{1}\left(\gamma_{1}^{* 2}-\gamma_{1}^{2}\right) h_{t}^{R}
$$

which is ultimately driven by $\lambda$.

Table 5 contains results from the joint estimation on returns, RV, and options. We estimate $E[\bar{h}]$ which then implies the $\omega$ parameters as before. In the GARV model we also estimate $\gamma_{1}^{*}$ and $\gamma_{2}^{*}$ along with $\gamma_{1}$ and $\gamma_{2}$, which together imply the risk premia $\lambda$ and $\chi$ from (6.4) above. In the ARV model we estimate $\gamma_{2}^{*}$ and $\gamma_{2}$ which imply $\chi$, and $\lambda$ is estimated as a free parameter. In the GARCH model, we estimate $\gamma_{1}$ and $\gamma_{1}^{*}$, which then imply $\lambda$.

The main results in Table 5 are as follows.

First, the likelihood based on returns and options strongly favors the GARV over ARV, with GARCH a distant third. The joint likelihood on Returns, RV, and Options strongly favors GARV over ARV. The GARV provides the best overall fit measured in terms of likelihood, and it also outperforms the other models based on option fit, as indicated by IVRMSE and VWRMSE. The pure ARV performs well compared with the pure GARCH in terms of overall fit.

Second, the persistence of volatility and the volatility components is largely in line with the 
results from Tables 1 and 3. The results for the GARV model are much closer to the option-based results in Table 3, in the sense that the persistence in return-based volatility is much higher in Table 5 than in Table 1.

Third, the estimated risk premia are very interesting, and indicate the challenges from fitting options and returns jointly. As discussed above, in the GARV model a variance risk premium can result from either $\lambda>0$ or $\chi<0$, or both. In the ARV model a positive variance premium is generated only if $\chi<0$. In the GARCH model, we need $\lambda>0$ to generate a positive risk premium. Table 5 shows that both risk prices contribute to the variance premium in GARV because $\lambda>0$ and $\chi<0$. Note the large gap between physical and risk-neutral unconditional volatility for the ARV and GARV models, which contrasts with the small gap in the GARCH model. The models' ability to generate this gap via $\chi$ is crucial when attempting to jointly fit options and returns.

Fourth, the improvement in option fit offered by the GARV model over the GARCH model, as measured by the IVRMSE and the VWRMSE, is very large. Note, in particular, that $\kappa$ is again small so that GARCH puts only little weight on the GARCH component. The ARV improvement on GARCH is small for VWRMSE but somewhat bigger for IVRMSE. Figure 9 shows that the superior fit of the GARV model is consistent across maturities, moneyness, and VIX levels, as was the case in Figure 7. Table 6 contains the numbers used in Figure 9.

\section{Alternative Model Specifications}

In this section we consider two extensions to the ARV and GARV models developed above.

The highly persistent RV dynamics in Figure 2 suggest that a two-component RV model may be warranted. We build such an extension to the ARV and GARV models in Section 7.1 below.

So far we have focused on affine variance dynamics so as to benefit from speedy computation of option prices via Fourier inversion. In Section 7.2 below we consider a more general non-affine modeling framework that nests our affine models as special cases.

\subsection{The Component RV Model}

Bates (2000) and Christoffersen, Heston and Jacobs (2009) find strong empirical support for twocomponent affine stochastic volatility models for S\&P500 index option valuation. ${ }^{11}$

Motivated by their findings, we now allow for more flexible RV dynamics by assuming that the

\footnotetext{
${ }^{11}$ See also Christoffersen, Jacobs, Ornthanalai, and Wang (2008), and Christoffersen, Dorion, Jacobs, and Wang (2010) for GARCH-based approaches.
} 
expected RV has two components

$$
E_{t}\left[R V_{t+1}\right] \equiv h_{t}^{R V}=q_{t}^{R V}+h_{t}^{R V}-q_{t}^{R V}
$$

where $q_{t}^{R V}$ denotes the long-run and $h_{t}^{R V}-q_{t}^{R V}$ the short run component. We further assume that the two components have the following autoregressive form

$$
\begin{aligned}
q_{t}^{R V} & =\omega_{L}+\kappa \alpha_{S} \gamma_{2}^{2} h_{t}^{R}+\beta_{L} q_{t-1}^{R V}+\alpha_{L}\left(\varepsilon_{2, t+1}-\gamma_{2} \sqrt{\bar{h}_{t}}\right)^{2} . \\
h_{t}^{R V}-q_{t}^{R V} & =\beta_{S}\left(h_{t-1}^{R V}-q_{t-1}^{R V}\right)+\alpha_{S} v\left(\varepsilon_{2, t+1}\right) .
\end{aligned}
$$

where $v\left(\varepsilon_{2, t+1}\right)$ is defined as in (2.9) above.

Using the GARV framework above, the complete specification of the new two-component model is provided by

$$
\begin{aligned}
R_{t+1} & =r+\lambda \bar{h}_{t}-\frac{1}{2} \bar{h}_{t}+\sqrt{\bar{h}_{t}} \varepsilon_{1, t+1}, \text { with } \\
\bar{h}_{t} & =\kappa h_{t}^{R}+(1-\kappa) h_{t}^{R V} \text {, where } \\
h_{t+1}^{R} & =\omega_{1}+\beta_{1} h_{t}^{R}+\alpha_{1}\left(\varepsilon_{1, t+1}-\gamma_{1} \sqrt{\bar{h}_{t}}\right)^{2}, \\
R V_{t+1} & =h_{t}^{R V}+\alpha_{2}\left[\left(\varepsilon_{2, t+1}-\gamma_{2} \sqrt{\bar{h}_{t}}\right)^{2}-\left(1+\gamma_{2}^{2} \bar{h}_{t}\right)\right] \\
h_{t}^{R V} & =q_{t}^{R V}+h_{t}^{R V}-q_{t}^{R V}, \text { and } \\
q_{t+1}^{R V} & =\omega_{L}+\kappa \alpha_{S} \gamma_{2}^{2} h_{t}^{R}+\beta_{L} q_{t}^{R V}+\alpha_{L}\left(\varepsilon_{2, t+1}-\gamma_{2} \sqrt{\bar{h}_{t}}\right)^{2} \\
h_{t+1}^{R V}-q_{t+1}^{R V} & =\beta_{S}\left(h_{t}^{R V}-q_{t}^{R V}\right)+\alpha_{S}\left[\left(\varepsilon_{2, t+1}-\gamma_{2} \sqrt{\bar{h}_{t}}\right)^{2}-\left(1+\gamma_{2}^{2} \bar{h}_{t}\right)\right]
\end{aligned}
$$

and we will refer to it as the two-component Generalized Affine RV model or GARV-2C for short.

We again allow for dependence between the $\varepsilon_{1, t+1}$ and $\varepsilon_{2, t+1}$ by assuming they follow a bivariate standard normal distribution with correlation $\rho$. A two-component model purely based on expected realized volatility emerges if $\kappa=0$. We will refer to this special case as the ARV-2C model.

The following constraints guarantee positive conditional variance

$$
\begin{aligned}
& \omega_{1} \geq 0, \beta_{1} \geq 0, \alpha_{1} \geq 0 \\
& \omega_{L} \geq \alpha_{S} \geq 0, \beta_{L} \geq \beta_{S} \geq(1-\kappa) \alpha_{S} \gamma_{2}^{2}, \alpha_{L} \geq 0 .
\end{aligned}
$$


To understand why these constraints work, it is useful the rewrite the dynamic of $h_{t+1}^{R V}$ as follows

$$
h_{t+1}^{R V}=\omega_{L}-\alpha_{S}+\left(\beta_{L}-\beta_{S}\right) q_{t}^{R V}+\left(\beta_{S}-(1-\kappa) \alpha_{S} \gamma_{2}^{2}\right) h_{t}^{R V}+\left(\alpha_{L}+\alpha_{S}\right)\left(\varepsilon_{2, t+1}-\gamma_{2} \sqrt{\bar{h}_{t}}\right)^{2}
$$

Note that the expected variance one day ahead is

$$
E_{t}\left(\bar{h}_{t+1}\right)=\kappa E_{t}\left(h_{t+1}^{R}\right)+(1-\kappa) E_{t}\left(h_{t+1}^{R V}\right)
$$

where

$$
\begin{aligned}
E_{t}\left(h_{t+1}^{R}\right)= & \omega_{1}+\alpha_{1}+\left(\beta_{1}+\kappa \alpha_{1} \gamma_{1}^{2}\right) h_{t}^{R} \\
& +(1-\kappa) \alpha_{1} \gamma_{1}^{2} q_{t}^{R V}+(1-\kappa) \alpha_{1} \gamma_{1}^{2}\left(h_{t}^{R V}-q_{t}^{R V}\right) \\
E_{t}\left(q_{t+1}^{R V}\right)= & \omega_{L}+\alpha_{L}+\kappa\left(\alpha_{S}+\alpha_{L}\right) \gamma_{2}^{2} h_{t}^{R} \\
& +\left(\beta_{L}+(1-\kappa) \alpha_{L} \gamma_{2}^{2}\right) q_{t}^{R V}+(1-\kappa) \alpha_{L} \gamma_{2}^{2}\left(h_{t}^{R V}-q_{t}^{R V}\right) \\
E_{t}\left(h_{t+1}^{R V}-q_{t+1}^{R V}\right)= & \beta_{S}\left(h_{t}^{R V}-q_{t}^{R V}\right) \\
E_{t}\left(h_{t+1}^{R V}\right)= & E_{t}\left(h_{t+1}^{R V}-q_{t+1}^{R V}\right)+E_{t}\left(q_{t+1}^{R V}\right) \\
= & \omega_{L}+\alpha_{L}+\kappa\left(\alpha_{S}+\alpha_{L}\right) \gamma_{2}^{2} h_{t}^{R} \\
& +\left(\beta_{L}+(1-\kappa) \alpha_{L} \gamma_{2}^{2}\right) q_{t}^{R V}+\left(\beta_{S}+(1-\kappa) \alpha_{L} \gamma_{2}^{2}\right)\left(h_{t}^{R V}-q_{t}^{R V}\right)
\end{aligned}
$$

Having three components in variance allows for potentially richer variance dynamics and richer term structures of variance, which is crucial for valuing options with maturities of several months. Appendix $\mathrm{C}$ contains details on the dynamic properties and on the risk neutralization of the GARV2C model.

Table 7 contains the results from the joint estimation of the ARV-2C and GARV-2C models on returns, RV and options. Comparing Table 7 with Table 5 we see that the GARV-2C model offers very little improvement over the GARV model in Table 5. This is perhaps not surprising as the GARV model already has two components: one from GARCH and one from RV. Note that the short run component in GARV-2C has a very low persistence and so contributes little to the modeling of the variance dynamic. This finding has interesting parallels to the results in Barndorff-Nielsen and Shephard (2002), who use RV to estimate stochastic volatility models with multiple components.

Table 7 also shows that the ARV-2C component model does offer a substantial improvement on the single component ARV model in Table 5. But the ARV-2C model is still outperformed by the GARV model in Table 5. 


\subsection{A Non-Affine Model}

Chernov, Gallant, Ghysels, and Tauchen (2003) find strong empirical support for non-affine stochastic volatility models when estimating on daily S\&P500 returns. Christoffersen, Jacobs and Mimouni (2010) confirm these results when assessing the S\&P500 index option fit of non-affine SV models.

Motivated by these papers we consider the following general class of non-affine RV-based models of returns and RV

$$
\begin{aligned}
R_{t+1} & =r+\lambda \bar{h}_{t}-\frac{1}{2} \bar{h}_{t}+\bar{h}_{t}^{1 / 2} z_{1, t+1} \\
h_{t+1}^{R} & =\omega_{1}+\beta_{1} h_{t}^{R}+\alpha_{1}\left(\left(h_{t}^{R}\right)^{\delta_{1}} z_{1, t+1}-\gamma_{1} \bar{h}_{t}^{1 / 2}\right)^{2} \\
h_{t+1}^{R V} & =\omega_{2}+\beta_{2} h_{t}^{R V}+\alpha_{2}\left(\left(h_{t}^{R V}\right)^{\delta_{2}} z_{2, t+1}-\gamma_{2} \bar{h}_{t}^{1 / 2}\right)^{2} \\
R V_{t+1} & =h_{t}^{R V}+\tilde{\alpha}_{2}\left[\left(\left(h_{t}^{R V}\right)^{\delta_{2}} z_{2, t+1}-\gamma_{2} \bar{h}_{t}^{1 / 2}\right)^{2}-\left(\left(h_{t}^{R V}\right)^{2 \delta_{2}}+\gamma_{2}^{2} \bar{h}_{t}\right)\right] \\
\bar{h}_{t} & =\kappa h_{t}^{R}+(1-\kappa) h_{t}^{R V}
\end{aligned}
$$

Note that our affine GARV model emerges as a special case when the new parameters, $\delta_{1}$ and $\delta_{2}$ are both zero. The affine GARV model of course nests the affine GARCH and ARV models as noted earlier. When $\kappa=1$ and $\delta_{1}=1 / 2$ in the new non-affine model, we get the non-affine NGARCH model from Engle and $\mathrm{Ng}$ (1993). When $\kappa=0$ and $\delta_{2}=1 / 2$ we get a new non-affine pure RV model which we refer to as NARV. A new generalized nonaffine model which we denote GNARV emerges when $\kappa$ is estimated freely and $\delta_{1}=\delta_{2}=1 / 2$.

While these non-affine models can be risk-neutralized using the approach taken above, option price calculations must be done by Monte Carlo and so are somewhat cumbersome in large samples such as ours. In Table 8 we therefore simply estimate the nonaffine models on returns and RV and compare the results with the affine models estimated in Table 1.

Table 8 shows that the likelihood values are considerably higher for non-affine models compared with the affine models in Table 1 . These findings confirm the results in the literature. ${ }^{12}$ More importantly, the results in Table 8 show that our main conclusion remains in the non-affine world: Non-affine GARV is preferred to non-affine GARCH when fitting returns. Incorporating the RV component thus helps in non-affine as well as affine settings.

The models in Table 8 fix $\delta_{1}$ and $\delta_{2}$ at $1 / 2$ but they could of course also be estimated as free parameters. When doing so preliminary evidence (not reported) suggests that the optimal values of

\footnotetext{
${ }^{12}$ For comparisons of affine and non-affine SV models, see Jones (2003) and Ait-Sahalia and Kimmel (2007). For comparisons of affine and non-affine GARCH models, see Hsieh and Ritchken (2005), and Christoffersen, Dorion, Jacobs and Wang (2010).
} 
$\delta_{1}$ and $\delta_{2}$ are very close to $1 / 2$. These results suggest that the models in Table 8 are well-specified and that developing fast and reliable numerical procedures for option valuation in these models would be a worthwhile pursuit. We leave this task for future work.

\section{Conclusion}

We develop a new affine discrete-time model that allows for option valuation in closed form. The model's volatility dynamic contains both a GARCH component and a realized volatility component. We find that incorporating realized volatility leads to a better fit on returns and realized volatility, and that it significantly reduces the pricing errors of the benchmark Heston-Nandi GARCH model. It is important to model expected realized volatility because the RV measures-while informative-are measured with error. Higher conditional moments for our newly proposed models are very different from those of the benchmark GARCH model.

We extend the benchmark model to allow for two components in the RV dynamic. We also briefly consider non-affine alternative specifications. The results suggest that developing quasi-closed form solutions for non-affine RV option models would be useful. Finally, we note that the leverage effect is modeled differently in the GARCH and RV dynamics. Exploring further the modeling of this important asymmetry is likely to yield substantial benefits.

\section{Appendix A. GARV Persistence and Stationarity}

In order to derive stationarity conditions in the GARV model, note that

$$
\begin{aligned}
& E_{t}\left[h_{t+1}^{R}\right]=\omega_{1}+\alpha_{1}+\left(\beta_{1}+\alpha_{1} \gamma_{1}^{2} \kappa\right) h_{t}^{R}+\alpha_{1} \gamma_{1}^{2}(1-\kappa) h_{t}^{R V} \\
& E_{t}\left[h_{t+1}^{R V}\right]=\tilde{\omega}_{2}+\tilde{\alpha}_{2}+\tilde{\alpha}_{2} \gamma_{2}^{2} \kappa h_{t}^{R}+\left(\tilde{\beta}_{2}+\tilde{\alpha}_{2} \gamma_{2}^{2}(1-\kappa)\right) h_{t}^{R V} .
\end{aligned}
$$

Therefore

$$
E_{t}\left(\begin{array}{c}
h_{t+1}^{R} \\
h_{t+1}^{R V}
\end{array}\right)=\phi_{0}+\phi_{1}\left(\begin{array}{c}
h_{t}^{R} \\
h_{t}^{R V}
\end{array}\right)
$$

where

$$
\begin{aligned}
\phi_{0} & =\left(\omega_{1}+\alpha_{1}, \tilde{\omega}_{2}+\tilde{\alpha}_{2}\right)^{\prime} \\
\phi_{1} & =\left[\begin{array}{cc}
\beta_{1}+\alpha_{1} \gamma_{1}^{2} \kappa & \alpha_{1} \gamma_{1}^{2}(1-\kappa) \\
\tilde{\alpha}_{2} \gamma_{2}^{2} \kappa & \tilde{\beta}_{2}+\tilde{\alpha}_{2} \gamma_{2}^{2}(1-\kappa)
\end{array}\right] .
\end{aligned}
$$


If the largest eigenvalue of $\phi_{1}$ has a modulus smaller than one we have

$$
E\left[\left(\begin{array}{c}
h_{t+1}^{R} \\
h_{t+1}^{R V}
\end{array}\right)\right]=\left(I_{2}-\phi_{1}\right)^{-1} \phi_{0}
$$

We can therefore derive the following necessary conditions for stationarity

$$
\begin{aligned}
\beta_{1} & <1, \quad \tilde{\beta}_{2}<1, \quad\left|\gamma_{1}\right|<\sqrt{\frac{1-\beta_{1}}{\alpha_{1} \kappa}} \\
\left|\gamma_{2}\right| & <\sqrt{\left(\frac{1-\tilde{\beta}_{2}}{1-\beta_{1}}\right)\left(\frac{1-\left(\beta_{1}+\alpha_{1} \gamma_{1}^{2} \kappa\right)}{\tilde{\alpha}_{2}(1-\kappa)}\right)}
\end{aligned}
$$

These conditions are imposed when estimating the models.

\section{Appendix B. Option Valuation}

In this appendix we first derive the moment generating function of the GARV model. We then show how to risk neutralize the model.

\section{B.1 The Moment Generating Function}

For the GARV model, using (2.4), (2.6) and (2.11) we have

$$
\begin{aligned}
& E_{t}\left[\exp \left(u R_{t+1}+w_{R} h_{t+1}^{R}+w_{R V} h_{t+1}^{R V}\right)\right]
\end{aligned}
$$

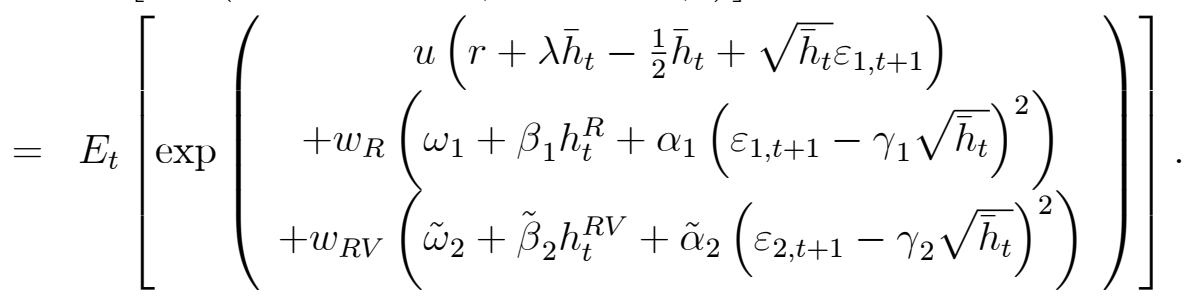

Rewriting, we get

$$
\begin{aligned}
& E_{t}\left[\exp \left(u R_{t+1}+w_{R} h_{t+1}^{R}+w_{R V} h_{t+1}^{R V}\right)\right] \\
= & \exp \left(u\left(r+\lambda \bar{h}_{t}-\frac{1}{2} \bar{h}_{t}\right)+w_{R}\left(\omega_{1}+\beta_{1} h_{t}^{R}\right)+w_{R V}\left(\tilde{\omega}_{2}+\tilde{\beta}_{2} h_{t}^{R V}\right)\right) \times \\
& E_{t}\left[\exp \left(u \sqrt{\bar{h}_{t}} \varepsilon_{1, t+1}+w_{R} \alpha_{1}\left(\varepsilon_{1, t+1}-\gamma_{1} \sqrt{\bar{h}_{t}}\right)^{2}+w_{R V} \tilde{\alpha}_{2}\left(\varepsilon_{2, t+1}-\gamma_{2} \sqrt{\bar{h}_{t}}\right)^{2}\right)\right] .
\end{aligned}
$$


Define $w_{1}=w_{R} \alpha_{1}$ and $w_{2}=w_{R V} \tilde{\alpha}_{2}$. We have

$$
\begin{aligned}
& E_{t}^{Q}\left[\exp \left(u \sqrt{\bar{h}_{t}} \varepsilon_{1, t+1}+w_{1}\left(\varepsilon_{1, t+1}-\gamma_{1} \sqrt{\bar{h}_{t}}\right)^{2}+w_{2}\left(\varepsilon_{2, t+1}-\gamma_{2} \sqrt{\bar{h}_{t}}\right)^{2}\right)\right] \\
& =E_{t}^{Q}\left[\exp \left(\begin{array}{c}
-\frac{1}{2} \ln \left(1-2 w_{2}\left(1-\rho^{2}\right)\right)+u \sqrt{\bar{h}_{t}} \varepsilon_{1, t+1} \\
+w_{1}\left(\varepsilon_{1, t+1}-\gamma_{1} \sqrt{\bar{h}_{t}}\right)^{2}+\frac{w_{2}\left(\rho \varepsilon_{1, t+1}-\gamma_{2} \sqrt{\bar{h}_{t}}\right)^{2}}{1-2 w_{2}\left(1-\rho^{2}\right)}
\end{array}\right)\right] \\
& =E_{t}^{Q}\left[\exp \left(-\frac{1}{2} \ln \left(1-2 w_{2}\left(1-\rho^{2}\right)\right)+a \varepsilon_{1, t+1}^{2}+b \sqrt{\bar{h}_{t}} \varepsilon_{1, t+1}+c \bar{h}_{t}\right)\right] \\
& =\exp \left(-\frac{1}{2} \ln \left(1-2 w_{2}\left(1-\rho^{2}\right)\right)-\frac{1}{2} \ln (1-2 a)+\left(c+\frac{b^{2}}{2(1-2 a)}\right) \bar{h}_{t}\right)
\end{aligned}
$$

with

$$
\begin{aligned}
a & =w_{1}+\frac{w_{2} \rho^{2}}{1-2 w_{2}\left(1-\rho^{2}\right)} \\
b & =u-2 \gamma_{1} w_{1}-\frac{2 \rho \gamma_{2} w_{2}}{1-2 w_{2}\left(1-\rho^{2}\right)} \\
c & =\left(\gamma_{1}\right)^{2} w_{1}+\frac{\left(\gamma_{2}\right)^{2} w_{2}}{1-2 w_{2}\left(1-\rho^{2}\right)}
\end{aligned}
$$

Collecting these results gives

$E_{t}\left[\exp \left(u R_{t+1}+w_{R} h_{t+1}^{R}+w_{R V} h_{t+1}^{R V}\right)\right]=\exp \left(A_{1}\left(u, w_{R}, w_{R V}\right) h_{t}^{R}+A_{2}\left(u, w_{R}, w_{R V}\right) h_{t}^{R V}+B\left(u, w_{R}, w_{R V}\right)\right)$

with

$$
\begin{aligned}
A_{1}\left(u, w_{R}, w_{R V}\right) & =\kappa\left[c+\frac{b^{2}}{2(1-2 a)}+u\left(\lambda-\frac{1}{2}\right)\right]+w_{R} \beta_{1} \\
A_{2}\left(u, w_{R}, w_{R V}\right) & =(1-\kappa)\left[c+\frac{b^{2}}{2(1-2 a)}+u\left(\lambda-\frac{1}{2}\right)\right]+w_{R V} \tilde{\beta}_{2} \\
B\left(u, w_{R}, w_{R V}\right) & =-\frac{1}{2} \ln \left(1-2 w_{2}\left(1-\rho^{2}\right)\right)-\frac{1}{2} \ln (1-2 a)+u r+w_{R} \omega_{1}+w_{R V} \tilde{\omega}_{2} .
\end{aligned}
$$

As the model is affine, we conjecture that the multi-period conditional moment generating function is of the form

$$
\begin{aligned}
\Psi_{t, t+M}(u) & \equiv E_{t}\left[\exp \left(u \sum_{j=1}^{M} R_{t+j}\right)\right] \\
& =\exp \left(C_{1}(u, M) h_{t}^{R}+C_{2}(u, M) h_{t}^{R V}+D(u, M)\right)
\end{aligned}
$$


Therefore

$$
\begin{aligned}
\Psi_{t, t+M+1}(u) & \equiv E_{t}\left[\exp \left(u \sum_{j=1}^{M+1} R_{t+j}\right)\right]=E_{t}\left[E_{t+1}\left[\exp \left(u \sum_{j=1}^{M+1} R_{t+j}\right)\right]\right] \\
& =E_{t}\left[\exp \left(u R_{t+1}\right) E_{t+1}\left[\exp \left(u \sum_{j=2}^{M+1} R_{t+j}\right)\right]\right] \\
& =E_{t}\left[\exp \left(u R_{t+1}\right) E_{t+1}\left[\exp \left(u \sum_{k=1}^{M} R_{t+1+k}\right)\right]\right] \\
& =E_{t}\left[\exp \left(u R_{t+1}+C_{1}(u, M) h_{t+1}^{R}+C_{2}(u, M) h_{t+1}^{R V}+D(u, M)\right)\right] \\
& =\exp \left(\begin{array}{c}
A_{1}\left(u, C_{1}(u, M), C_{2}(u, M)\right) h_{t}+A_{2}\left(u, C_{1}(u, M), C_{2}(u, M)\right) h_{t}^{R V} \\
+B\left(u, C_{1}(u, M), C_{2}(u, M)\right)+D(u, M)
\end{array}\right) .
\end{aligned}
$$

This yields

$$
\begin{aligned}
C_{1}(u, M+1) & =A_{1}\left(u, C_{1}(u, M), C_{2}(u, M)\right) \\
C_{2}(u, M+1) & =A_{2}\left(u, C_{1}(u, M), C_{2}(u, M)\right) \\
D(u, M+1) & =B\left(u, C_{1}(u, M), C_{2}(u, M)\right)+D(u, M),
\end{aligned}
$$

with the following initial conditions

$$
\begin{aligned}
C_{1}(u, 1) & =A_{1}(u, 0,0) \\
C_{2}(u, 1) & =A_{2}(u, 0,0) \\
D(u, 1) & =B(u, 0,0) .
\end{aligned}
$$

\section{B.2 Risk Neutralization}

We follow the risk-neutralization approach of Christoffersen, Elkamhi, Feunou and Jacobs (2010). The GARV model has two shocks, and so we rely on the following pricing kernel

$$
Z_{t+1}=\frac{\exp \left(\nu_{1, t} \varepsilon_{1, t+1}+\nu_{2, t} \varepsilon_{2, t+1}\right)}{E_{t}\left[\exp \left(\nu_{1, t} \varepsilon_{1, t+1}+\nu_{2, t} \varepsilon_{2, t+1}\right)\right]}
$$

Given that $\varepsilon_{1, t+1}$ and $\varepsilon_{2, t+1}$ are bivariate standard normal with correlation $\rho$ we have

$$
Z_{t+1}=\exp \left(\nu_{1, t} \varepsilon_{1, t+1}+\nu_{2, t} \varepsilon_{2, t+1}-\frac{\nu_{1, t}^{2}}{2}-\frac{\nu_{2, t}^{2}}{2}-\nu_{1, t} \nu_{2, t} \rho\right) .
$$


We need to impose that

$$
E_{t}^{Q}\left[\exp \left(R_{t+1}\right)\right]=\exp (r)
$$

The risk-neutral expected compound return is

$$
E_{t}^{Q}\left[\exp \left(R_{t+1}\right)\right]=E_{t}\left[Z_{t+1} \exp \left(R_{t+1}\right)\right]=\exp \left(r+\lambda \bar{h}_{t}+\left(\nu_{1, t}+\nu_{2, t} \rho\right) \sqrt{\bar{h}_{t}}\right)
$$

Setting this equal to the risk-free rate gives the condition

$$
\begin{gathered}
E_{t}^{Q}\left[\exp \left(R_{t+1}\right)\right]=\exp (r) \\
\Leftrightarrow \lambda \bar{h}_{t}+\left(\nu_{1, t}+\nu_{2, t} \rho\right) \sqrt{\bar{h}_{t}}=0 \\
\Leftrightarrow \nu_{1, t}+\nu_{2, t} \rho=-\lambda \sqrt{\bar{h}_{t}} .
\end{gathered}
$$

For the bivariate shocks we have the risk-neutral expectation

$$
\begin{aligned}
E_{t}^{Q}\left[\exp \left(u_{1} \varepsilon_{1, t+1}+u_{2} \varepsilon_{2, t+1}\right)\right] & =E_{t}\left[Z_{t+1} \exp \left(u_{1} \varepsilon_{1, t+1}+u_{2} \varepsilon_{2, t+1}\right)\right] \\
& =\exp \left(u_{1}\left(\nu_{1, t}+\nu_{2, t} \rho\right)+u_{2}\left(\nu_{2, t}+\nu_{1, t} \rho\right)+\frac{u_{1}^{2}}{2}+\frac{u_{2}^{2}}{2}+u_{1} u_{2} \rho\right)
\end{aligned}
$$

Following Christoffersen, Elkamhi, Feunou and Jacobs (2010), under the risk-neutral probability measure we have

$$
\begin{aligned}
& \varepsilon_{1, t+1}^{*}=\varepsilon_{1, t+1}-\left(\nu_{1, t}+\nu_{2, t} \rho\right) \\
& \varepsilon_{2, t+1}^{*}=\varepsilon_{2, t+1}-\left(\nu_{2, t}+\nu_{1, t} \rho\right)
\end{aligned}
$$

These innovations are bivariate standard normal with correlation $\rho$. We can therefore rewrite the model as follows

$$
\begin{aligned}
R_{t+1} & =r+\lambda \bar{h}_{t}-\frac{1}{2} \bar{h}_{t}+\sqrt{\bar{h}_{t}} \varepsilon_{1, t+1} \\
& =r+\lambda \bar{h}_{t}-\frac{1}{2} \bar{h}_{t}+\sqrt{\bar{h}_{t}}\left(\varepsilon_{1, t+1}^{*}+\nu_{1, t}+\nu_{2, t} \rho\right) \\
& =r+\lambda \bar{h}_{t}+\sqrt{\bar{h}_{t}}\left(\nu_{1, t}+\nu_{2, t} \rho\right)-\frac{1}{2} \bar{h}_{t}+\sqrt{\bar{h}_{t}} \varepsilon_{1, t+1}^{*} \\
& =r-\frac{1}{2} \bar{h}_{t}+\sqrt{\bar{h}_{t}} \varepsilon_{1, t+1}^{*}
\end{aligned}
$$


which holds from (B.5) because

$$
\lambda \bar{h}_{t}+\sqrt{\bar{h}_{t}}\left(\nu_{1, t}+\nu_{2, t} \rho\right)=0 .
$$

Using (B.6) and (B.5), the dynamic of the GARCH component of the volatility can be rewritten in term of the risk-neutral shock as follows

$$
\begin{aligned}
h_{t+1}^{R} & =\omega_{1}+\beta_{1} h_{t}^{R}+\alpha_{1}\left(\varepsilon_{1, t+1}-\gamma_{1} \sqrt{\bar{h}_{t}}\right)^{2} \\
& =\omega_{1}+\beta_{1} h_{t}^{R}+\alpha_{1}\left(\varepsilon_{1, t+1}^{*}-\gamma_{1}^{*} \sqrt{\bar{h}_{t}}\right)^{2}
\end{aligned}
$$

with $\gamma_{1}^{*}=\gamma_{1}+\lambda$.

Now recall the dynamics of the realized variance $R V_{t+1}$

$$
\begin{aligned}
R V_{t+1} & =h_{t}^{R V}+\alpha_{2}\left[\left(\varepsilon_{2, t+1}-\gamma_{2} \sqrt{\bar{h}_{t}}\right)^{2}-\left(1+\gamma_{2}^{2} \bar{h}_{t}\right)\right] \\
& =h_{t}^{R V}+\alpha_{2}\left[\left(\varepsilon_{2, t+1}^{*}+\nu_{2, t}+\nu_{1, t} \rho-\gamma_{2}{\sqrt{h_{t}}}^{2}-\left(1+\gamma_{2}^{2} \bar{h}_{t}\right)\right]\right. \\
& =h_{t}^{R V}+\alpha_{2}\left[\left(\varepsilon_{2, t+1}^{*}-\gamma_{2 t}^{*} \sqrt{\bar{h}_{t}}\right)^{2}-\left(1+\gamma_{2}^{2} \bar{h}_{t}\right)\right]
\end{aligned}
$$

with

$$
\gamma_{2 t}^{*}=\gamma_{2}-\frac{\nu_{2, t}+\nu_{1, t} \rho}{\sqrt{\bar{h}_{t}}} .
$$

In order to keep the model affine under $Q$, we will impose that $\gamma_{2 t}^{*}$ is constant. This can be done via the condition

$$
\nu_{2, t}+\nu_{1, t} \rho=\chi \sqrt{\bar{h}_{t}}
$$

where $\chi$ is a constant risk premium parameter to be estimated. We then have

$$
\nu_{2, t}=\chi \sqrt{\bar{h}_{t}}-\nu_{1, t} \rho
$$

and thus

$$
\gamma_{2 t}^{*}=\gamma_{2}-\chi .
$$


We now have

$$
\begin{aligned}
R V_{t+1} & =h_{t}^{R V}+\alpha_{2}\left[\left(\varepsilon_{2, t+1}^{*}-\gamma_{2}^{*} \sqrt{\bar{h}_{t}}\right)^{2}-\left(1+\gamma_{2}^{2} \bar{h}_{t}\right)\right] \\
& =h_{t}^{R V}+\alpha_{2}\left(\gamma_{2}^{* 2}-\gamma_{2}^{2}\right) \bar{h}_{t}+\alpha_{2}\left[\left(\varepsilon_{2, t+1}^{*}-\gamma_{2}^{*} \sqrt{\bar{h}}_{t}\right)^{2}-\left(1+\gamma_{2}^{* 2} \bar{h}_{t}\right)\right]
\end{aligned}
$$

Note that

$$
\begin{aligned}
E_{t}^{Q}\left[R V_{t+1}\right] & \equiv h_{t}^{R V *} \\
& =h_{t}^{R V}+\alpha_{2}\left(\gamma_{2}^{* 2}-\gamma_{2}^{2}\right) \bar{h}_{t} \\
& =E_{t}\left[R V_{t+1}\right]+\alpha_{2}\left(\gamma_{2}^{* 2}-\gamma_{2}^{2}\right) \bar{h}_{t},
\end{aligned}
$$

Finally, using (B.6) and (B.7) we obtain

$$
h_{t+1}^{R V}=\tilde{\omega}_{2}+\tilde{\beta}_{2} h_{t}^{R V}+\tilde{\alpha}_{2}\left(\varepsilon_{2, t+1}^{*}-\gamma_{2}^{*}{\sqrt{\bar{h}_{t}}}^{2}\right.
$$

In summary, the dynamic under the risk-neutral probability measure is

$$
\begin{aligned}
R_{t+1} & =r-\frac{1}{2} \bar{h}_{t}+\sqrt{\bar{h}_{t}} \varepsilon_{1, t+1}^{*} \\
\bar{h}_{t} & =\kappa h_{t}^{R}+(1-\kappa) h_{t}^{R V}
\end{aligned}
$$

with

$$
\begin{aligned}
h_{t+1}^{R} & =\omega_{1}+\beta_{1} h_{t}^{R}+\alpha_{1}\left(\varepsilon_{1, t+1}^{*}-\gamma_{1}^{*} \sqrt{\bar{h}_{t}}\right)^{2} \\
R V_{t+1} & =h_{t}^{R V *}+\alpha_{2}\left[\left(\varepsilon_{2, t+1}^{*}-\gamma_{2}^{*} \sqrt{\bar{h}_{t}}\right)^{2}-\left(1+\gamma_{2}^{* 2} \bar{h}_{t}\right)\right] \\
h_{t+1}^{R V} & =\tilde{\omega}_{2}+\tilde{\beta}_{2} h_{t}^{R V}+\tilde{\alpha}_{2}\left(\varepsilon_{2, t+1}^{*}-\gamma_{2}^{*} \sqrt{\bar{h}_{t}}\right)^{2} \\
h_{t}^{R V *} & =h_{t}^{R V}+\alpha_{2}\left(\gamma_{2}^{* 2}-\gamma_{2}^{2}\right) \bar{h}_{t},
\end{aligned}
$$

where $\varepsilon_{1, t+1}^{*}$ and $\varepsilon_{2, t+1}^{*}$, are bivariate standard normal with correlation $\rho$ under $Q$.

\section{Appendix C. The Component Model}

In this appendix we present various results on the two-component models. 


\section{C.1 Stationarity and Unconditional Moments}

The short run RV-component is covariance stationary if and only if $\beta_{S}<1$, which implies that $E\left[h_{t}^{R V}-q_{t}^{R V}\right]=0$. Given the stationarity of the short run component, both the GARCH component and the long run RV-component are covariance stationary if and only if the largest eigenvalue of the following matrix is less than one in absolute value.

$$
\phi=\left[\begin{array}{cc}
\beta_{1}+\kappa \alpha_{1} \gamma_{1}^{2} & (1-\kappa) \alpha_{1} \gamma_{1}^{2} \\
\kappa\left(\alpha_{S}+\alpha_{L}\right) \gamma_{2}^{2} & \beta_{L}+(1-\kappa) \alpha_{L} \gamma_{2}^{2}
\end{array}\right] .
$$

This is equivalent to

$$
\begin{aligned}
\beta_{1}+\kappa \alpha_{1} \gamma_{1}^{2} & <1 \\
\beta_{L}+(1-\kappa) \alpha_{L} \gamma_{2}^{2} & <1 \\
\kappa(1-\kappa) \alpha_{1}\left(\alpha_{S}+\alpha_{L}\right) \gamma_{1}^{2} \gamma_{2}^{2} & <\left(1-\left(\beta_{1}+\kappa \alpha_{1} \gamma_{1}^{2}\right)\right)\left(1-\left(\beta_{L}+(1-\kappa) \alpha_{L} \gamma_{2}^{2}\right)\right)
\end{aligned}
$$

These conditions are imposed when estimating the model.

\section{C.2 The Risk-Neutral Model}

We assume the pricing kernel from Appendix B.2. Following the same steps as above, we can show that returns and volatility components have the following dynamics under the risk-neutral probability measure

$$
\begin{aligned}
& R_{t+1}=r-\frac{1}{2} \bar{h}_{t}+\sqrt{\bar{h}_{t}} \varepsilon_{1, t+1}^{*} \\
& h_{t+1}^{R}=\omega_{1}+\beta_{1} h_{t}^{R}+\alpha_{1}\left(\varepsilon_{1, t+1}^{*}-\gamma_{1}^{*} \sqrt{\bar{h}_{t}}\right)^{2}
\end{aligned}
$$

with $\gamma_{1}^{*}=\gamma_{1}+\lambda$.

$$
\begin{aligned}
R V_{t+1} & =h_{t}^{R V}+\alpha_{2}\left[\left(\varepsilon_{2, t+1}^{*}-\gamma_{2}^{*} \sqrt{\bar{h}_{t}}\right)^{2}-\left(1+\gamma_{2}^{2} \bar{h}_{t}\right)\right] \\
& =h_{t}^{R V}+\alpha_{2}\left(\gamma_{2}^{* 2}-\gamma_{2}^{2}\right) \bar{h}_{t}+\alpha_{2}\left[\left(\varepsilon_{2, t+1}^{*}-\gamma_{2}^{*} \sqrt{\bar{h}_{t}}\right)^{2}-\left(1+\gamma_{2}^{* 2} \bar{h}_{t}\right)\right]
\end{aligned}
$$


with $\gamma_{2}^{*}=\gamma_{2}-\chi$.

$$
\begin{aligned}
& q_{t}^{R V}=\omega_{L}+\kappa \alpha_{S} \gamma_{2}^{2} h_{t}^{R}+\beta_{L} q_{t-1}^{R V}+\alpha_{L}\left[\frac{R V_{t+1}-h_{t}^{R V}}{\alpha_{2}}+\left(1+\gamma_{2}^{2} \bar{h}_{t}\right)\right] . \\
&=\omega_{L}+\kappa \alpha_{S} \gamma_{2}^{2} h_{t}^{R}+\beta_{L} q_{t}^{R V}+\alpha_{L}\left(\varepsilon_{2, t+1}^{*}-\gamma_{2}^{*} \sqrt{\bar{h}_{t}}\right)^{2} . \\
& h_{t+1}^{R V}-q_{t+1}^{R V}=\beta_{S}\left(h_{t-1}^{R V}-q_{t-1}^{R V}\right)+\alpha_{S}\left[\left(\varepsilon_{2, t+1}^{*}-\gamma_{2}^{*} \sqrt{\bar{h}_{t}}\right)^{2}-\left(1+\gamma_{2}^{2} \bar{h}_{t}\right)\right]
\end{aligned}
$$

\section{C.3 The Moment Generating Function}

Similarly to the case of the GARV model, we need the moment generating function of the component model in order to derive the closed-form option price formula. This section provides the derivation of this moment generating function. The assumption that $\varepsilon_{1, t+1}^{*} \stackrel{Q}{\sim} N(0,1)$ implies that

$$
E_{t}^{Q}\left[\exp \left(a \varepsilon_{1, t+1}^{* 2}+b \sqrt{\bar{h}_{t}} \varepsilon_{1, t+1}^{*}+c \bar{h}_{t}\right)\right]=\exp \left(-\frac{1}{2} \ln (1-2 a)+\left(c+\frac{b^{2}}{2(1-2 a)}\right) \bar{h}_{t}\right)
$$

We therefore get

$$
\begin{aligned}
& E_{t}^{Q}\left[\exp \left(u R_{t+1}+w_{R} h_{t+1}^{R}+w_{R V, L} q_{t+1}^{R V}+w_{R V, S}\left(h_{t+1}^{R V}-q_{t+1}^{R V}\right)\right)\right] \\
& =\exp \left(\begin{array}{c}
u\left(r-\frac{1}{2} \bar{h}_{t}\right)+w_{R}\left(\omega_{1}+\beta_{1} h_{t}^{R}\right)+w_{R V, L}\left(\omega_{L}+\kappa \alpha_{S} \gamma_{2}^{2} h_{t}^{R}+\beta_{L} q_{t}^{R V}\right) \\
+w_{R V, S} \beta_{S}\left(h_{t}^{R V}-q_{t}^{R V}\right)-w_{R V, S} \alpha_{S}\left(1+\gamma_{2}^{2} \bar{h}_{t}\right)
\end{array}\right) \times \\
& E_{t}^{Q}\left[\exp \left(u \sqrt{\bar{h}_{t}} \varepsilon_{1, t+1}^{*}+w_{1}\left(\varepsilon_{1, t+1}-\gamma_{1}^{*} \sqrt{\bar{h}_{t}}\right)^{2}+w_{2}\left(\varepsilon_{2, t+1}-\gamma_{2}^{*} \sqrt{\bar{h}_{t}}\right)^{2}\right)\right]
\end{aligned}
$$

where we have defined $w_{1}=w_{R} \alpha_{1}$ and $w_{2}=w_{R V, L} \alpha_{L}+w_{R V, S} \alpha_{S}$. We have

$$
\begin{aligned}
& E_{t}^{Q}\left[\exp \left(u \sqrt{\bar{h}_{t}} \varepsilon_{1, t+1}^{*}+w_{1}\left(\varepsilon_{1, t+1}-\gamma_{1}^{*} \sqrt{\bar{h}_{t}}\right)^{2}+w_{2}\left(\varepsilon_{2, t+1}-\gamma_{2}^{*} \sqrt{\bar{h}_{t}}\right)^{2}\right)\right] \\
& =E_{t}^{Q}\left[\exp \left(\begin{array}{c}
-\frac{1}{2} \ln \left(1-2 w_{2}\left(1-\rho^{2}\right)\right)+u \sqrt{\bar{h}_{t}} \varepsilon_{1, t+1}^{*} \\
+w_{1}\left(\varepsilon_{1, t+1}-\gamma_{1}^{*} \sqrt{\bar{h}_{t}}\right)^{2}+\frac{w_{2}\left(\rho \varepsilon_{1, t+1}^{*}-\gamma_{2}^{*} \sqrt{\bar{h}_{t}}\right)^{2}}{1-2 w_{2}\left(1-\rho^{2}\right)}
\end{array}\right)\right] \\
& =E_{t}^{Q}\left[\exp \left(-\frac{1}{2} \ln \left(1-2 w_{2}\left(1-\rho^{2}\right)\right)+a \varepsilon_{1, t+1}^{* 2}+b \sqrt{\bar{h}_{t}} \varepsilon_{1, t+1}^{*}+c \bar{h}_{t}\right)\right] \\
& =\exp \left(-\frac{1}{2} \ln \left(1-2 w_{2}\left(1-\rho^{2}\right)\right)-\frac{1}{2} \ln (1-2 a)+\left(c+\frac{b^{2}}{2(1-2 a)}\right) \bar{h}_{t}\right)
\end{aligned}
$$


where

$$
\begin{aligned}
& a=w_{1}+\frac{w_{2} \rho^{2}}{1-2 w_{2}\left(1-\rho^{2}\right)}, \quad b=u-2 \gamma_{1}^{*} w_{1}-\frac{2 \rho \gamma_{2}^{*} w_{2}}{1-2 w_{2}\left(1-\rho^{2}\right)} \\
& c=\left(\gamma_{1}^{*}\right)^{2} w_{1}+\frac{\left(\gamma_{2}^{*}\right)^{2} w_{2}}{1-2 w_{2}\left(1-\rho^{2}\right)}, \quad d=c+\frac{b^{2}}{2(1-2 a)}-\frac{1}{2} u-w_{R V, S} \alpha_{S} \gamma_{2}^{2}
\end{aligned}
$$

Collecting terms, we get

$$
\begin{aligned}
E_{t}^{Q}\left[\exp \left(u R_{t+1}+w_{R} h_{t+1}^{R}+w_{R V, L} q_{t+1}^{R V}+w_{R V, S}\left(h_{t+1}^{R V}-q_{t+1}^{R V}\right)\right)\right] \\
=\exp \left(\begin{array}{c}
u r+w_{R} \omega_{1}+w_{R V, L} \omega_{L}-w_{R V, S} \alpha_{S}-\frac{1}{2} \ln \left(1-2 w_{2}\left(1-\rho^{2}\right)\right)-\frac{1}{2} \ln (1-2 a) \\
+\left(w_{R} \beta_{1}+w_{R V, L} \kappa \alpha_{S} \gamma_{2}^{2}+\kappa d\right) h_{t}^{R}+\left(w_{R V, L} \beta_{L}+(1-\kappa) d\right) q_{t}^{R V} \\
+\left(w_{R V, S} \beta_{S}+(1-\kappa) d\right)\left(h_{t}^{R V}-q_{t}^{R V}\right)
\end{array}\right)
\end{aligned}
$$




\section{References}

[1] Aït-Sahalia, Y., and J. Jacod (2009), Analyzing the Spectrum of Asset Returns: Jump and Volatility Components in High Frequency Data, Journal of Economic Literature, forthcoming.

[2] Aït-Sahalia, Y., and R. Kimmel (2007), Maximum Likelihood Estimation of Stochastic Volatility Models, Journal of Financial Economics, 83, 413-452.

[3] Aït-Sahalia, Y., P. Mykland, and L. Zhang (2005), How Often to Sample a Continuous-Time Process in the Presence of Market Microstructure Noise, Review of Financial Studies 18, 351416.

[4] Andersen, T.G., and T. Bollerslev (1998), Answering the Skeptics: Yes, Standard Volatility Models Do Provide Accurate Forecasts, International Economic Review 39, 885-905.

[5] Andersen, T.G., T. Bollerslev, F.X. Diebold, and P. Labys (2003), Modeling and Forecasting Realized Volatility, Econometrica 71, 579-625.

[6] Andrews, D. (1993), Tests for Parameter Instability and Structural Change With Unknown Change Point, Econometrica 61, 821-856.

[7] Andrews, D. (2001), Testing When a Parameter is on the Boundary of the Maintained Hypothesis, Econometrica 69, 683-734.

[8] Andrews, D. and W. Ploberger (1994), Optimal Tests when a Nuisance Parameter is Present Only Under the Alternative, Econometrica 62, 1383-1414.

[9] Barndorff-Nielsen, O.E., and N. Shephard (2002), Econometric Analysis of Realized Volatility and its Use in Estimating Stochastic Volatility Models, Journal of the Royal Statistical Society B 64, 253-280.

[10] Barone-Adesi, G., R. Engle, and L. Mancini (2008), A GARCH Option Pricing Model with Filtered Historical Simulation, Review of Financial Studies 21, 1223-1258.

[11] Bates, D. (1996), Testing Option Pricing Models, in Handbook of Statistics, Statistical Methods in Finance, G.S. Maddala and C.R. Rao (eds.), pp. 567-611. Amsterdam: Elsevier.

[12] Bates, D. (2000), Post-87 Crash Fears in S\&P 500 Futures Options, Journal of Econometrics 94, 181-238. 
[13] Bollerslev, T. (1986), Generalized Autoregressive Conditional Heteroskedasticity, Journal of Econometrics 31, 307-327.

[14] Bollerslev, T., U. Kretschmer, C. Pigorsch, and G. Tauchen (2009), A Discrete-Time Model for Daily S\&P 500 Returns and Realized Variations: Jumps and Leverage Effects, Journal of Econometrics 150, 151-166.

[15] Bollerslev, T. and H. Mikkelsen (1999), Long-Term Equity AnticiPation Securities and Stock Market Volatility Dynamics, Journal of Econometrics 92, 75-99.

[16] Bollerslev, T. and J.M. Wooldridge (1992), Quasi-Maximum Likelihood Estimation and Inference in Dynamic Models with Time-Varying Covariances, Econometric Reviews 11, 143-172.

[17] Chen, X., E. Ghysels, and F. Wang (2011), Hybrid-GARCH Models and Intra-daily Return Periodicity, Journal of Time Series Econometrics, forthcoming.

[18] Chernov, M., A.R. Gallant, E. Ghysels, and G. Tauchen (2003), Alternative Models for Stock Price Dynamics, Journal of Econometrics 116, 225-257.

[19] Cho, S. J. and H. White (2007), Testing for Regime Switching, Econometrica 75, 1671-1720.

[20] Christoffersen, P., C. Dorion, K. Jacobs, and Y. Wang (2010), Volatility Components, Affine Restrictions and Non-Normal Innovations, Journal of Business and Economic Statistics 28, 486-502.

[21] Christoffersen, P., R. Elkamhi, B. Feunou, and K. Jacobs (2010), Option Valuation with Conditional Heteroskedasticity and Non-Normality, Review of Financial Studies, 23, 2139-2183.

[22] Christoffersen, P., S. Heston, and K. Jacobs (2009), The Shape and Term Structure of the Index Option Smirk: Why Multifactor Stochastic Volatility Models Work so Well, Management Science 55, 1914-1932.

[23] Christoffersen, P., K. Jacobs and K. Mimouni (2010), Volatility Dynamics for the S\&P500: Evidence from Realized Volatility, Daily Returns and Option Prices, Review of Financial Studies $23,3141-3189$.

[24] Christoffersen, P., K. Jacobs, C. Ornthanalai, and Y. Wang (2008), Option Valuation with Long-run and Short-run Volatility Components, Journal of Financial Economics 90, 272-297.

[25] Corsi, F., N. Fusari, and D. La Vecchia (2009), Back to History: Pricing Options with Realized Volatility, Working Paper, University of Lugano. 
[26] Duan, J.C. (1995), The GARCH Option Pricing Model, Mathematical Finance 5, 13-32.

[27] Engle, R. (1982), Autoregressive Conditional Heteroskedasticity with Estimates of the Variance of UK Inflation, Econometrica 50, 987-1008.

[28] Engle, R. (2002), New Frontiers in ARCH Models, Journal of Applied Econometrics 17, 425446.

[29] Engle, R., and G. Gallo (2006), A Multiple Indicators Model for Volatility using Intra-daily Data, Journal of Econometrics 131, 3-27.

[30] Engle, R., and C. Mustafa (1992), Implied ARCH Models from Options Prices, Journal of Econometrics 52, 289-311.

[31] Engle, R., and V. Ng (2003), Measuring and Testing the Impact of News on Volatility, Journal of Finance 48, 1749-1778.

[32] Forsberg, L., and T. Bollerslev (2002), Bridging the Gap Between the Distribution of Realized (ECU) and ARCH Modelling (of the Euro): The GARCH-NIG Model, Journal of Applied Econometrics 17, 535-548.

[33] French, K., G.W. Schwert, and R. Stambaugh (1987), Expected Stock Returns and Volatility, Journal of Financial Economics 19, 3-30.

[34] Glosten, L., R. Jagannathan, and D. Runkle (1993), On the Relation between the Expected Value and the Volatility of the Nominal Excess Return on Stocks, Journal of Finance 48, 1779-1801.

[35] Gourieroux, C., A. Monfort, and A. Trognon (1984), Pseudo Maximum Likelihood Methods: Theory, Econometrica 52, 681-700.

[36] Hansen, P., Z. Huang, and H. Shek (2011), Realized GARCH: A Joint Model for Returns and Realized Measures of Volatility, Journal of Applied Econometrics, forthcoming.

[37] Hansen, P., and A. Lunde (2005), A Realized Variance for the Whole Day Based on Intermittent High-Frequency Data, Journal of Financial Econometrics 3, 525-554.

[38] Heston, S., and S. Nandi (2000), A Closed-Form GARCH Option Pricing Model, Review of Financial Studies 13, 585-626. 
[39] Hsieh, K. and P. Ritchken (2005), An Empirical Comparison of GARCH Option Pricing Models, Review of Derivatives Research 8, 129-150.

[40] Jones, C. (2003), The Dynamics of Stochastic Volatility: Evidence from Underlying and Options Markets, Journal of Econometrics 116, 181-224.

[41] Nelson, D. B. (1991), Conditional Heteroskedasticity in Asset Returns: A New Approach, Econometrica 59, 347-370.

[42] Renault, E. (1997), Econometric Models of Option Pricing Errors, in Advances in Economics and Econometrics, Seventh World Congress, D.M. Kreps and K.F. Wallis (eds.), Econometric Society Monographs, Cambridge University Press, pp. 223-278.

[43] Ritchken, P., and R. Trevor (1999), Pricing Options Under Generalized GARCH and Stochastic Volatility Processes, Journal of Finance 54, 377-402.

[44] Shephard, N., and K. Sheppard (2010), Realising the Future: Forecasting with High-FrequencyBased Volatility (Heavy) models, Journal of Applied Econometrics 25, 197-231.

[45] Stentoft, L. (2008), Option Pricing Using Realized Volatility, CREATES Research Paper No. 2008-13.

[46] Trolle, A., and E. Schwartz (2009), Unspanned Stochastic Volatility and the Pricing of Commodity Derivatives, Review of Financial Studies 22, 4423-4461.

[47] Zhang, L., P. Mykland, and Y. Aitt-Sahalia (2005), A Tale of Two Time Scales: Determining Integrated Volatility with Noisy High-Frequency Data, Journal of the American Statistical Association 100, 1394-1411. 
Figure 1: Daily Futures Returns and Daily Realized Volatility from Intraday Returns. 1990-2010.
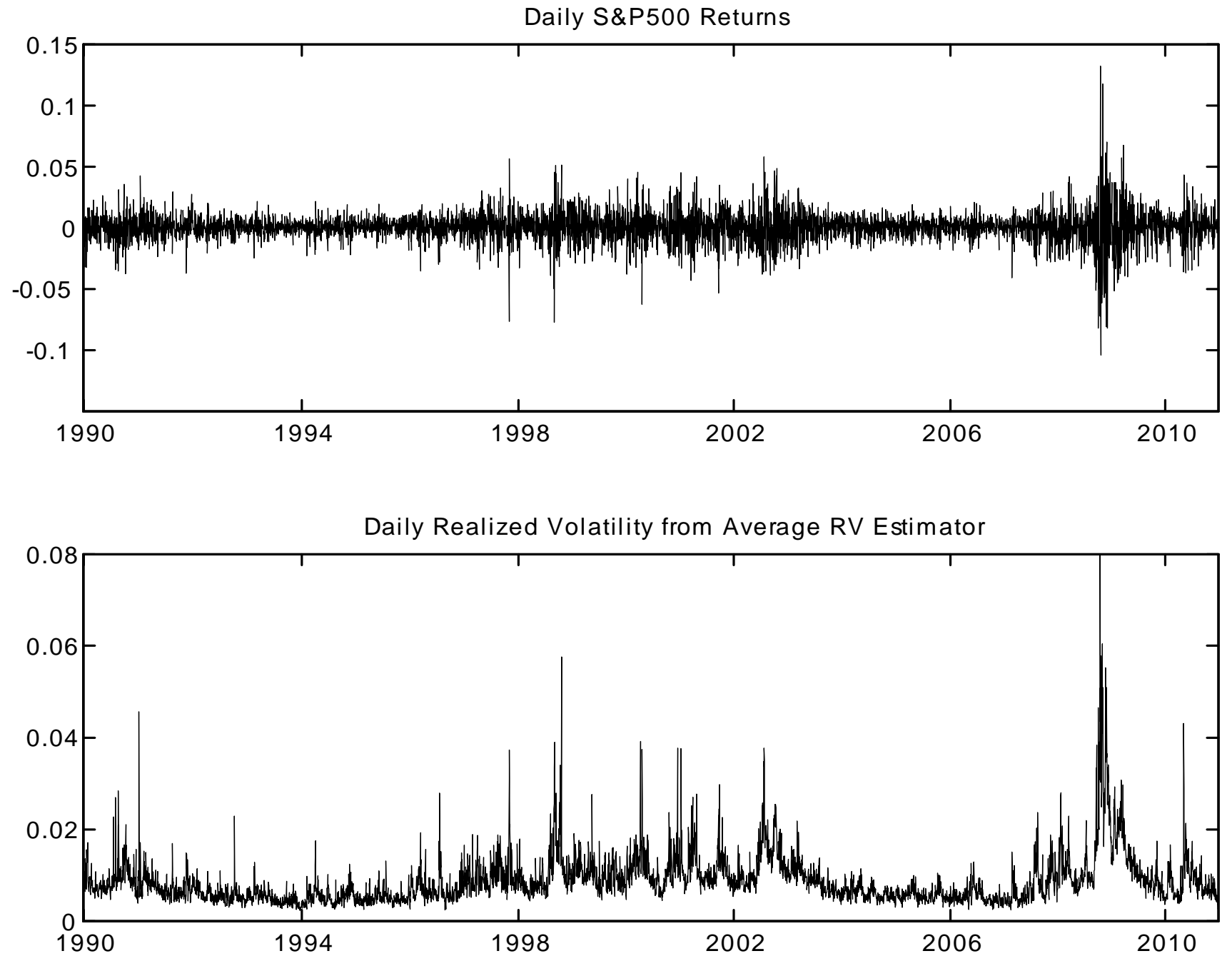

Notes to Figure: In the top panel we plot the daily returns on the S\&P500 index futures from January 2, 1990 to December 31, 2010. In the bottom panel we plot the square root of the realized variance using the Average RV estimator which is computed daily using 5-minute squared returns on a grid of 1-minute intraday index futures prices. The realized variance measure has been rescaled to match the unconditional variance of daily returns. 
Figure 2: Autocorrelations of Returns and Realized Variance
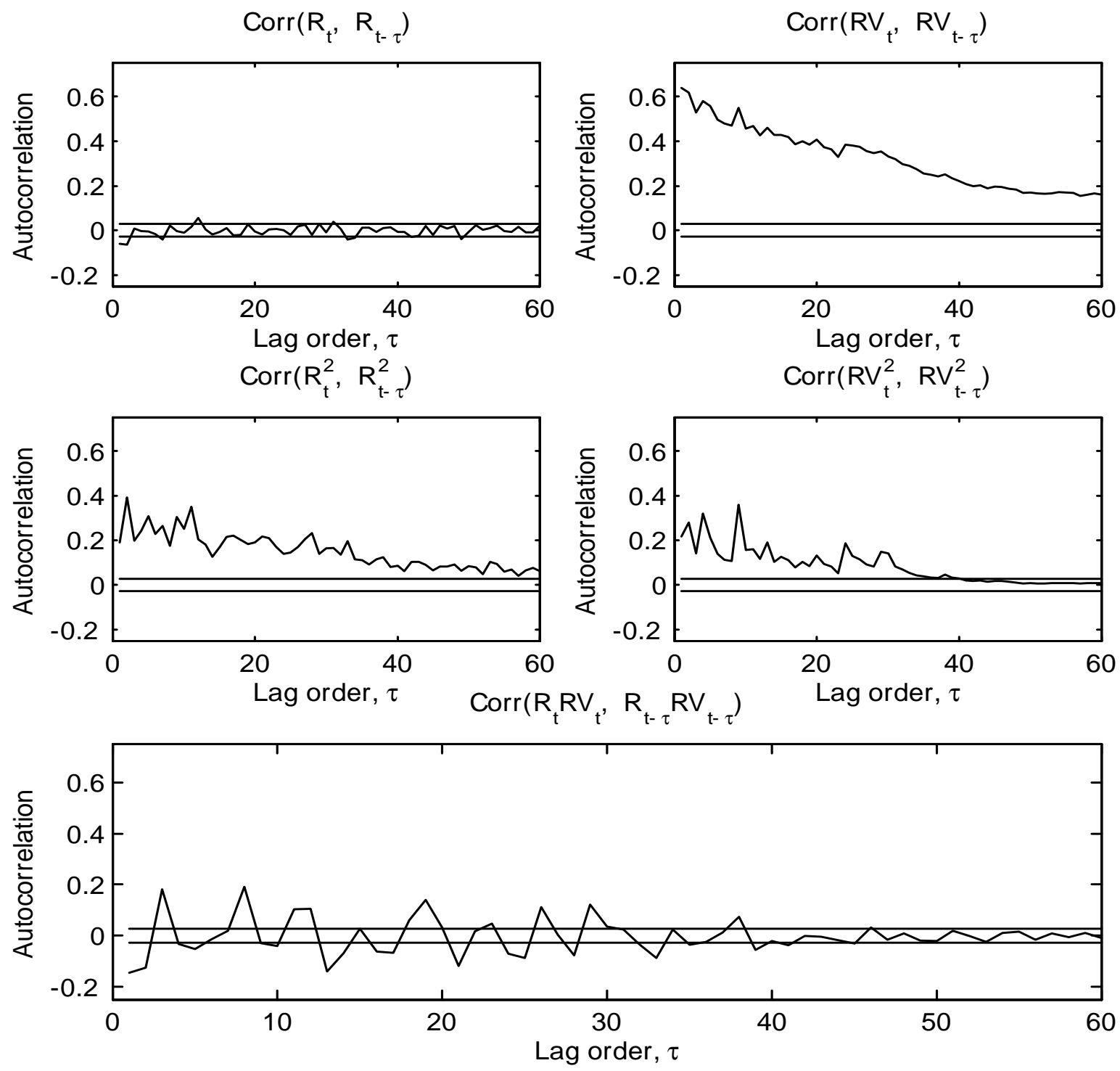

Notes to Figure: We plot the autocorrelations of returns, $R_{t}$, squared returns, $R_{t}^{2}$, realized variance, $R V_{t}$, squared realized variance, $R V_{t}^{2}$, and the product of returns and realized variance, $R_{t} R V_{t}$. We use lag orders between 1 and 60 trading days in each plot. The horizontal lines denote Bartlett $+/$ - two-standard-error bands. 
Figure 3: Autocorrelations of GARV Model Residuals
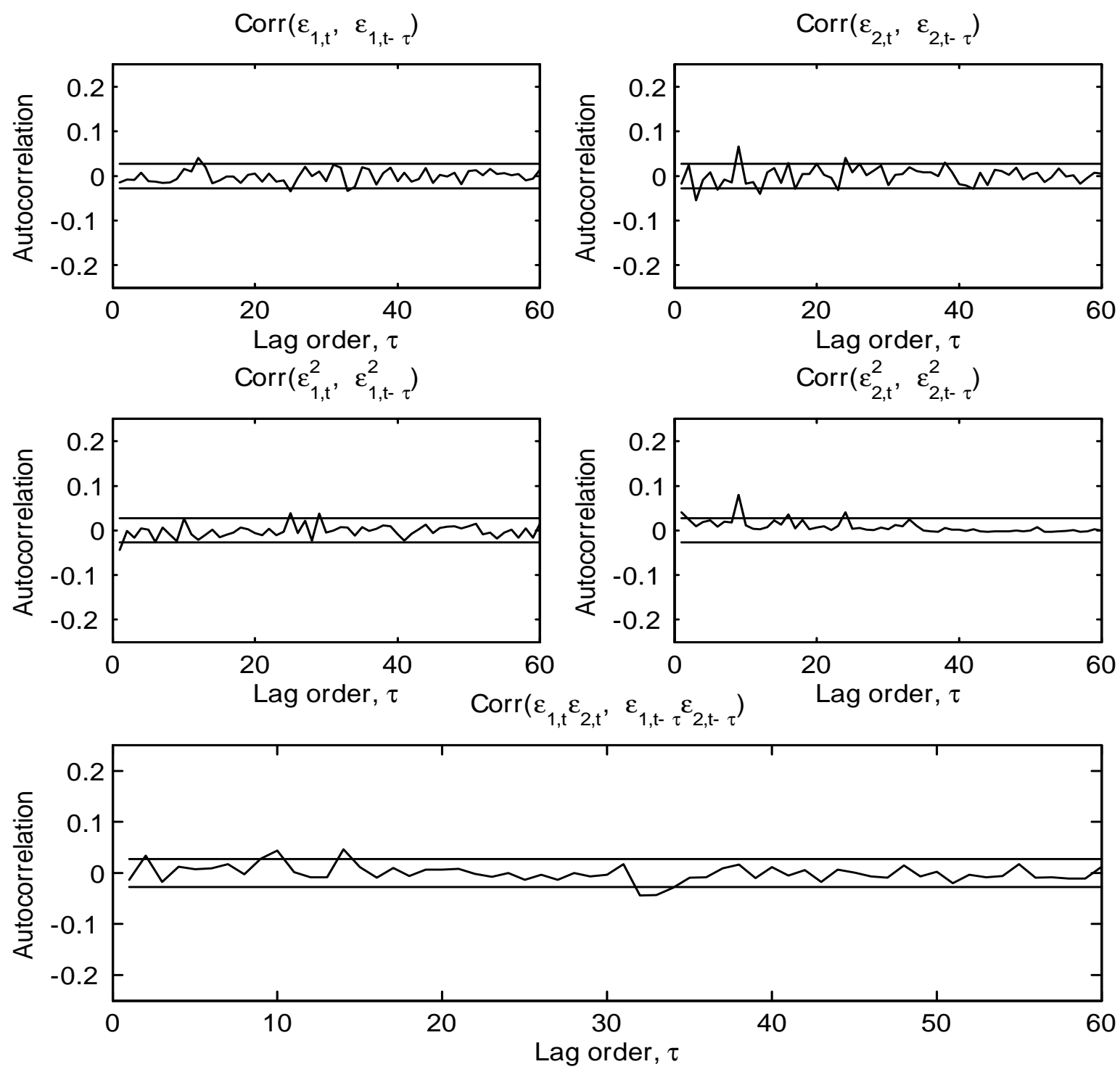

Notes to Figure: Using the GARV model estimated in Table 1, we plot the autocorrelations of the return residual, $\varepsilon_{1, t}$, the squared return residual, $\varepsilon_{1, t}^{2}$, the realized variance residual, $\varepsilon_{2, t}$, the squared realized variance residual, $\varepsilon_{2, t}^{2}$, and the product of the return and realized variance residuals, $\varepsilon_{1, t} \varepsilon_{2, t}$. The horizontal lines denote Bartlett +/- two-standard-error bands. 
Figure 4: Daily Conditional Volatility, 1990-2010.

Models Estimated on Returns and RV.

GARCH Model

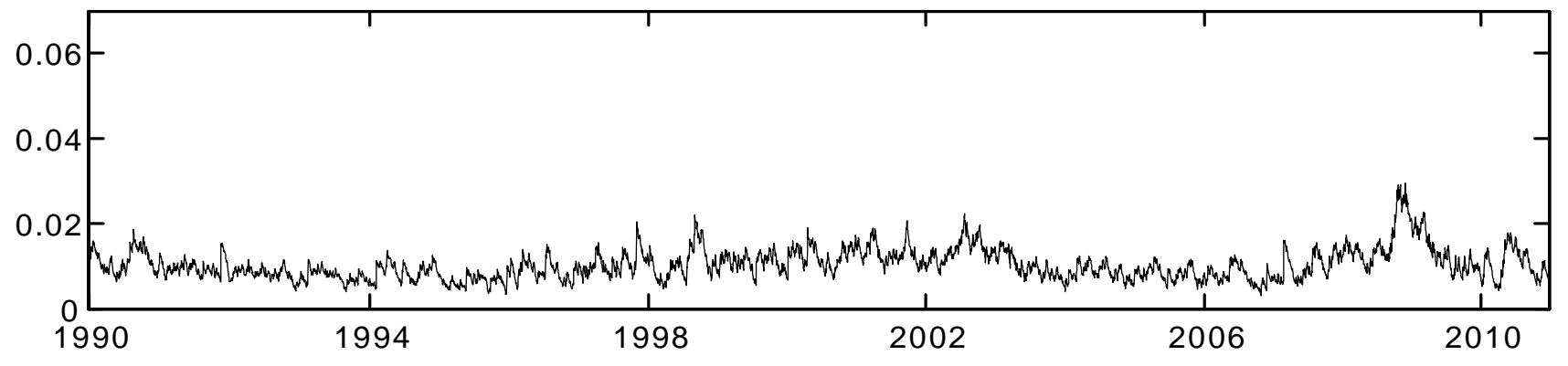

ARV Model

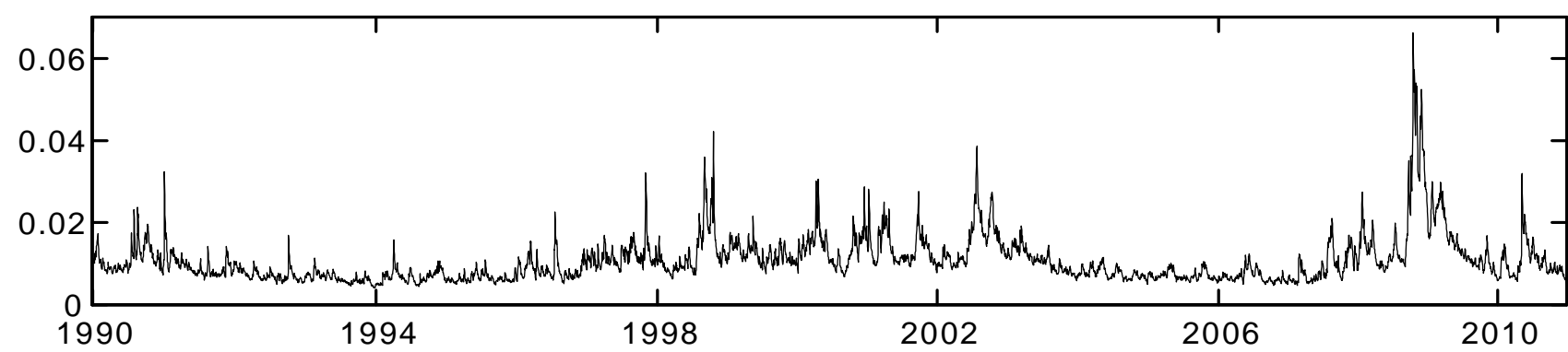

GARV Model

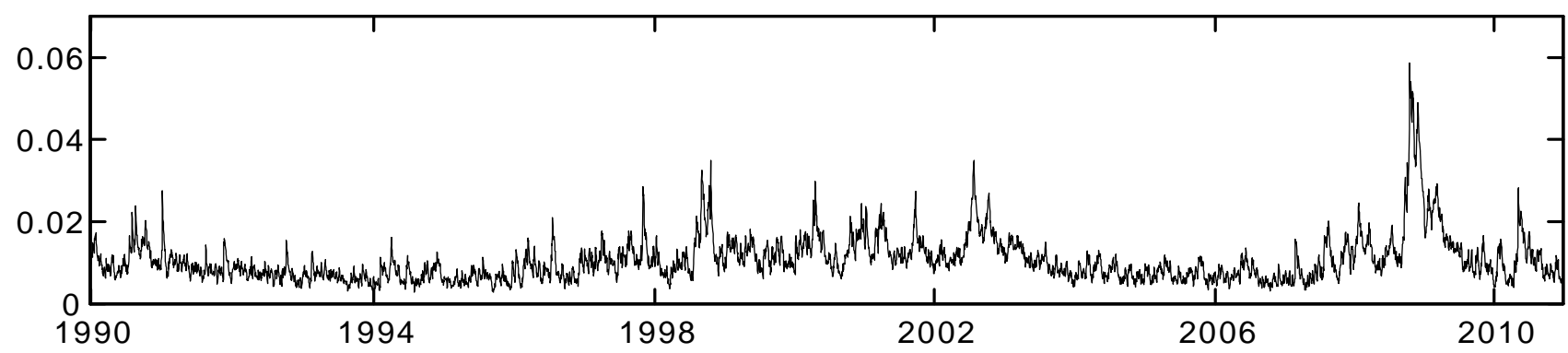

Notes to Figure: For the three models we investigate, we plot the daily conditional volatility, $\sqrt{\bar{h}_{t}}$, from January 2, 1990 to December 31, 2010. The top panel shows the GARCH model, the middle panel the ARV model, and the bottom panel the GARV model. The model parameters are from Table 1. 
Figure 5: Daily Conditional Volatility of Variance, 1990-2010.

Models Estimated on Returns and RV.
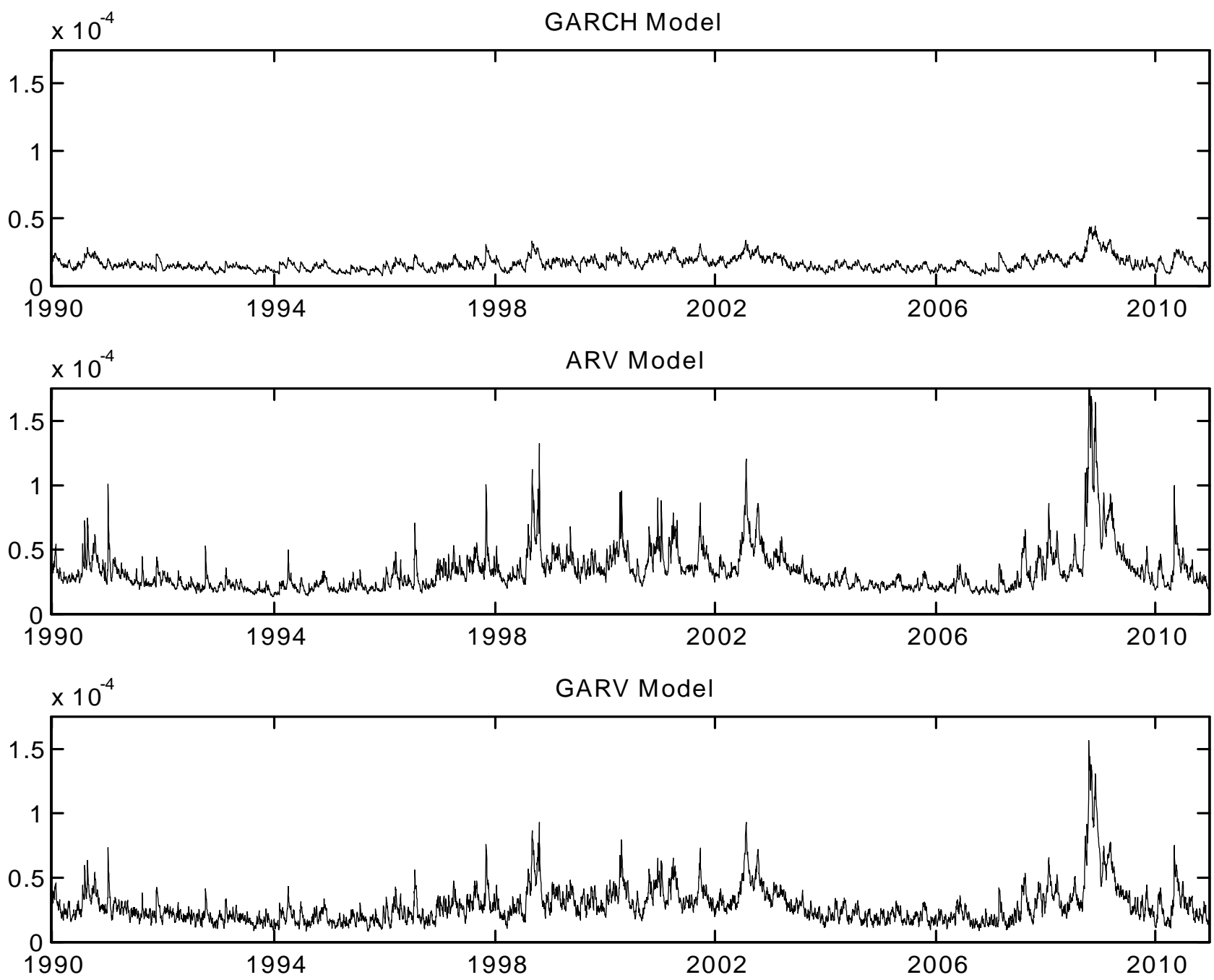

Notes to Figure: For the three models we investigate, we plot the daily conditional volatility of variance, $\sqrt{\operatorname{Var}_{t}\left(\bar{h}_{t+1}\right)}$, from January 2, 1990 to December 31, 2010. The top panel shows the GARCH model, the middle panel the ARV model, and the bottom panel the GARV model. The model parameters are from Table 1. 
Figure 6: Daily Conditional Correlation of Return and Variance, 1990-2010.

Models Estimated on Returns and RV.

GARCH Model

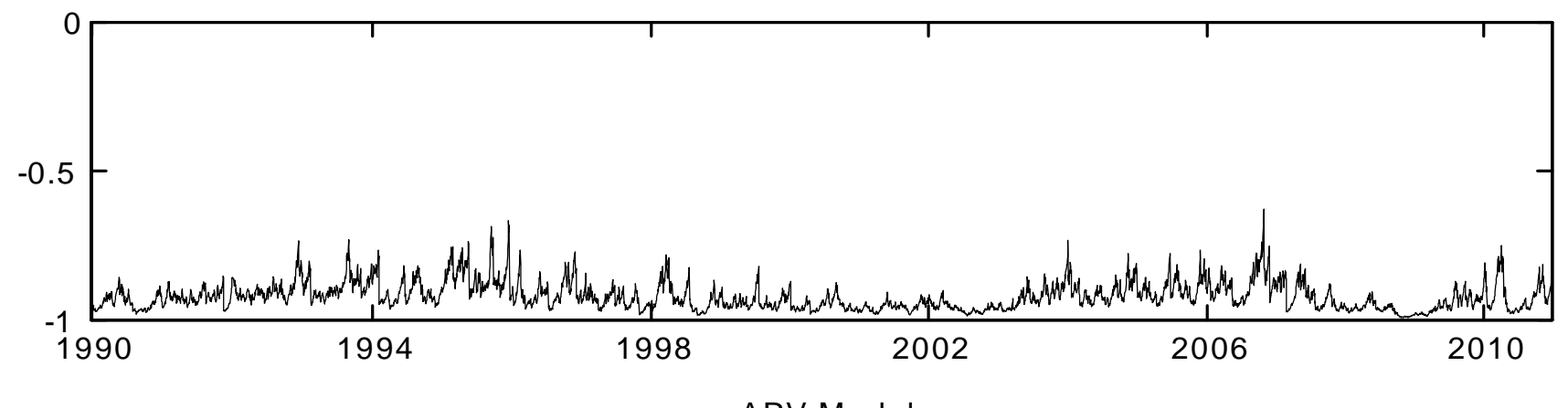

ARV Model

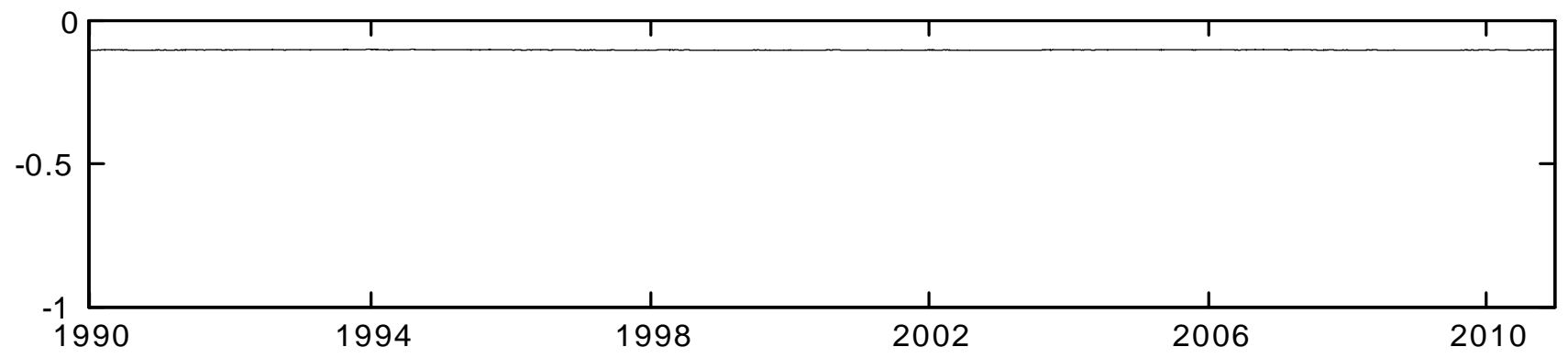

GARV Model

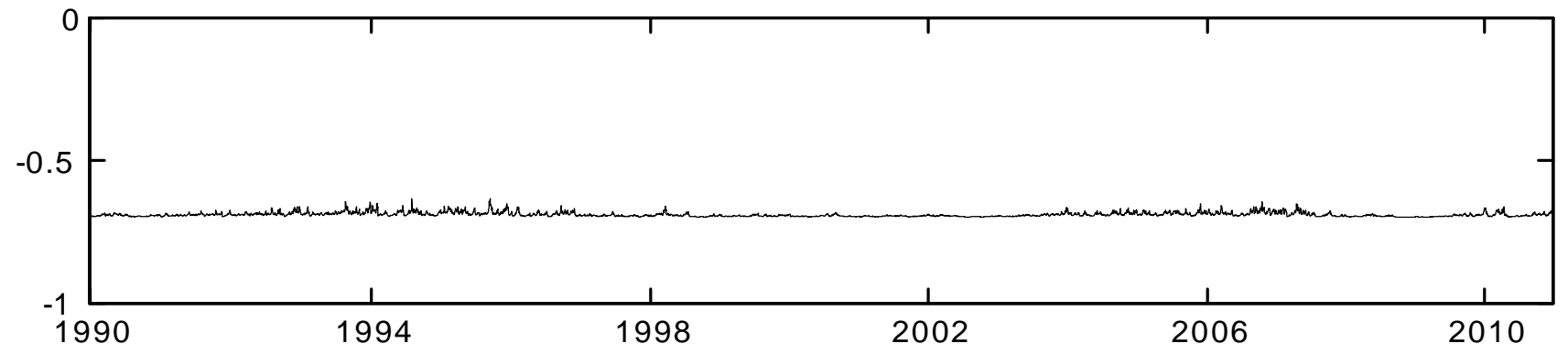

Notes to Figure: For the three models we investigate, we plot the daily conditional correlation between return and variance, $\operatorname{Corr}_{t}\left(R_{t+1}, \bar{h}_{t+1}\right)$, from January 2, 1990 to December 31, 2010. The top panel shows the GARCH model, the middle panel the ARV model, and the bottom panel the GARV model. The model parameters are from Table 1. 
Figure 7: IVRMSE by Moneyness, Maturity and VIX Level, 1996-2009.

Models Estimated on Options. Volatility is Filtered on Returns and RV.
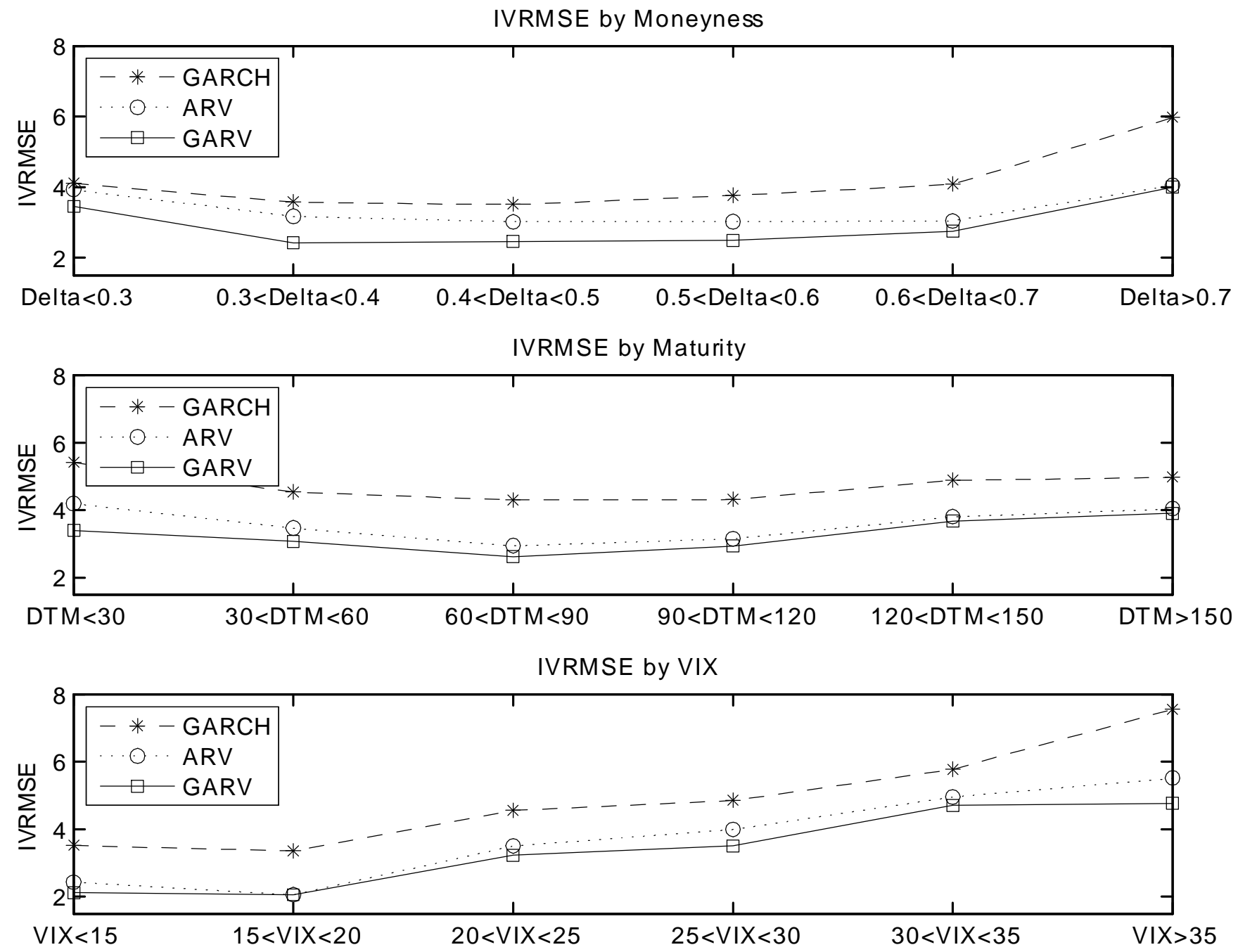

Notes to Figure: For each of the three models we investigate we plot the implied volatility root mean squared error (IVRMSE) separated into six moneyness bins (top panel), six maturity bins (middle panel), and six bins by VIX level (bottom panel). The dashed line with $*$ markers corresponds to the GARCH model, the dotted line with o markers corresponds to the ARV model, and the solid line with $\square$ markers corresponds to the GARV model. The model parameters are from Table 3. 
Figure 8: Weekly IVRMSE from ATM Options, 1996-2009.

Models Estimated on Options. Volatility is Filtered on Returns and RV.
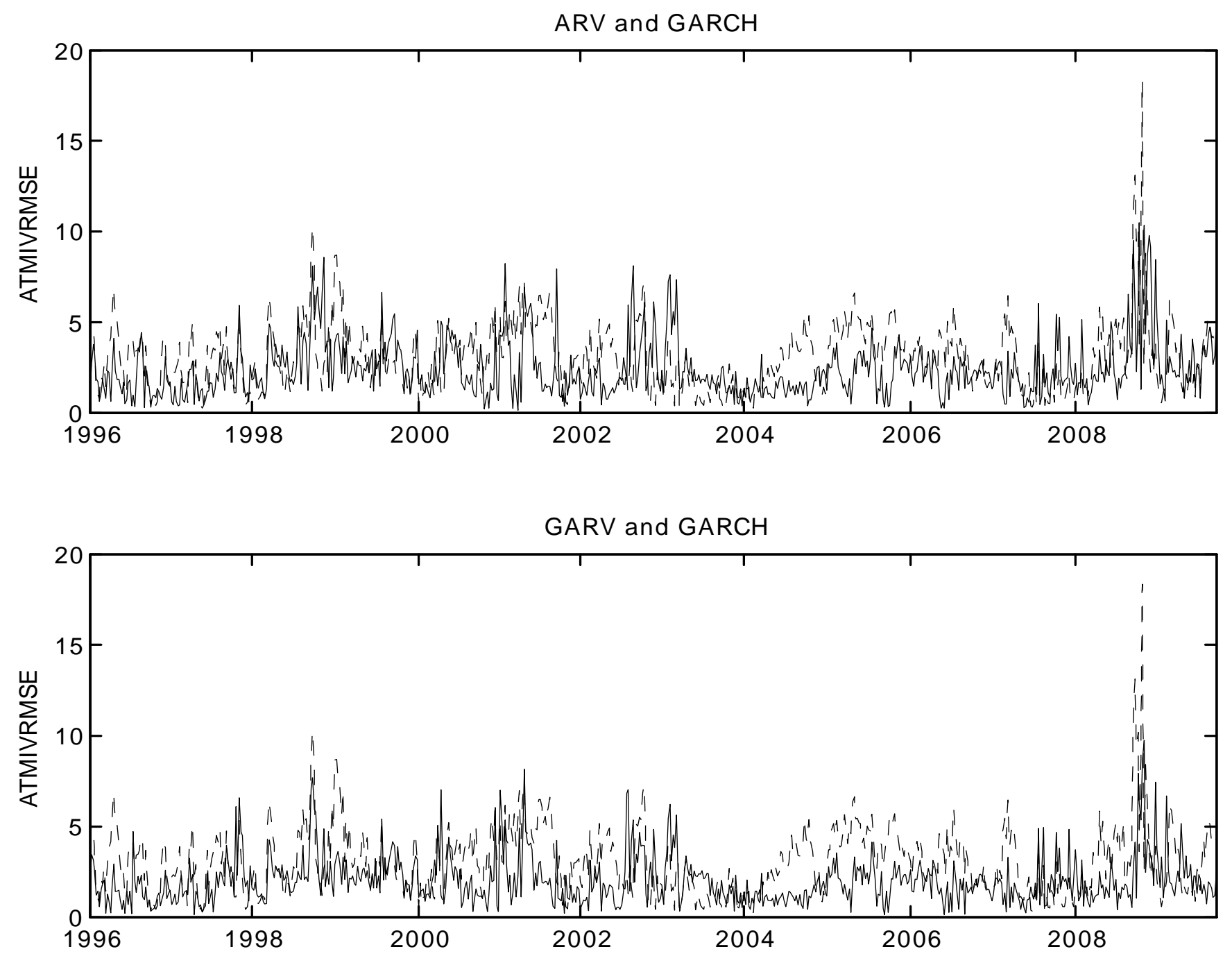

Notes to Figure: On each Wednesday we plot the implied volatility root mean squared error (IVRMSE) using at-the-money (ATM) options. The solid line in each panel corresponds to an RV-based model, and the dashed line corresponds to the GARCH model. The top panel contains the ARV model and the bottom panels contains the GARV model. The model parameters are from Table 3. 
Figure 9: IVRMSE by Moneyness, Maturity and VIX Level, 1996-2009.

Models Estimated Jointly on Returns, RV, and Options.
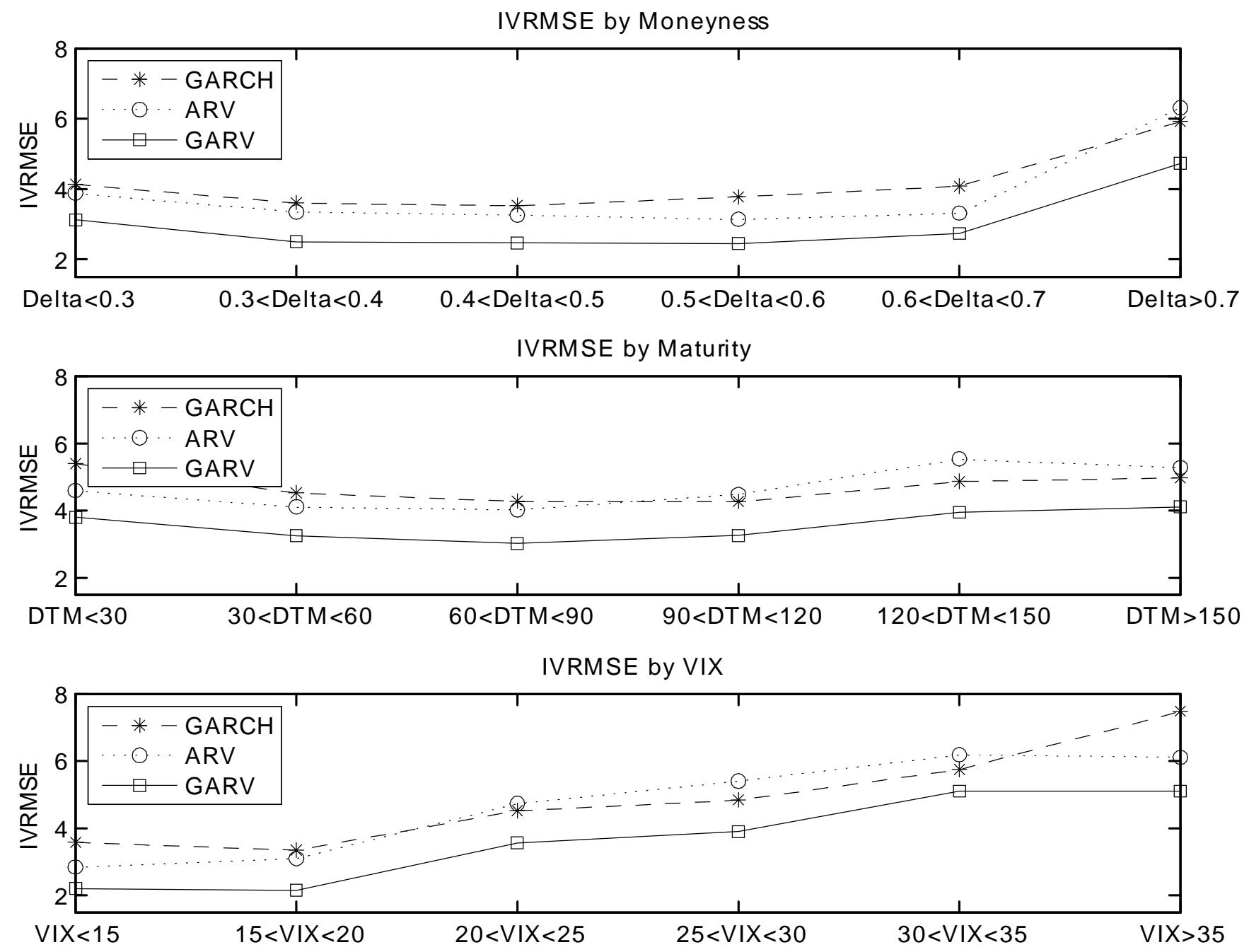

Notes to Figure: For each of the three models we investigate we plot the implied volatility root mean squared error (IVRMSE) separated into six moneyness bins (top panel), six maturity bins (middle panel), and six bins by VIX level (bottom panel). The dashed line with * markers corresponds to the GARCH model, the dotted line with o markers corresponds to the ARV model, and the solid line with $\square$ markers corresponds to the GARV model. The model parameters are from Table 5 . 
Figure 10: Weekly IVRMSE from ATM Options, 1996-2009.

Models Estimated Jointly on Returns, RV and Options.
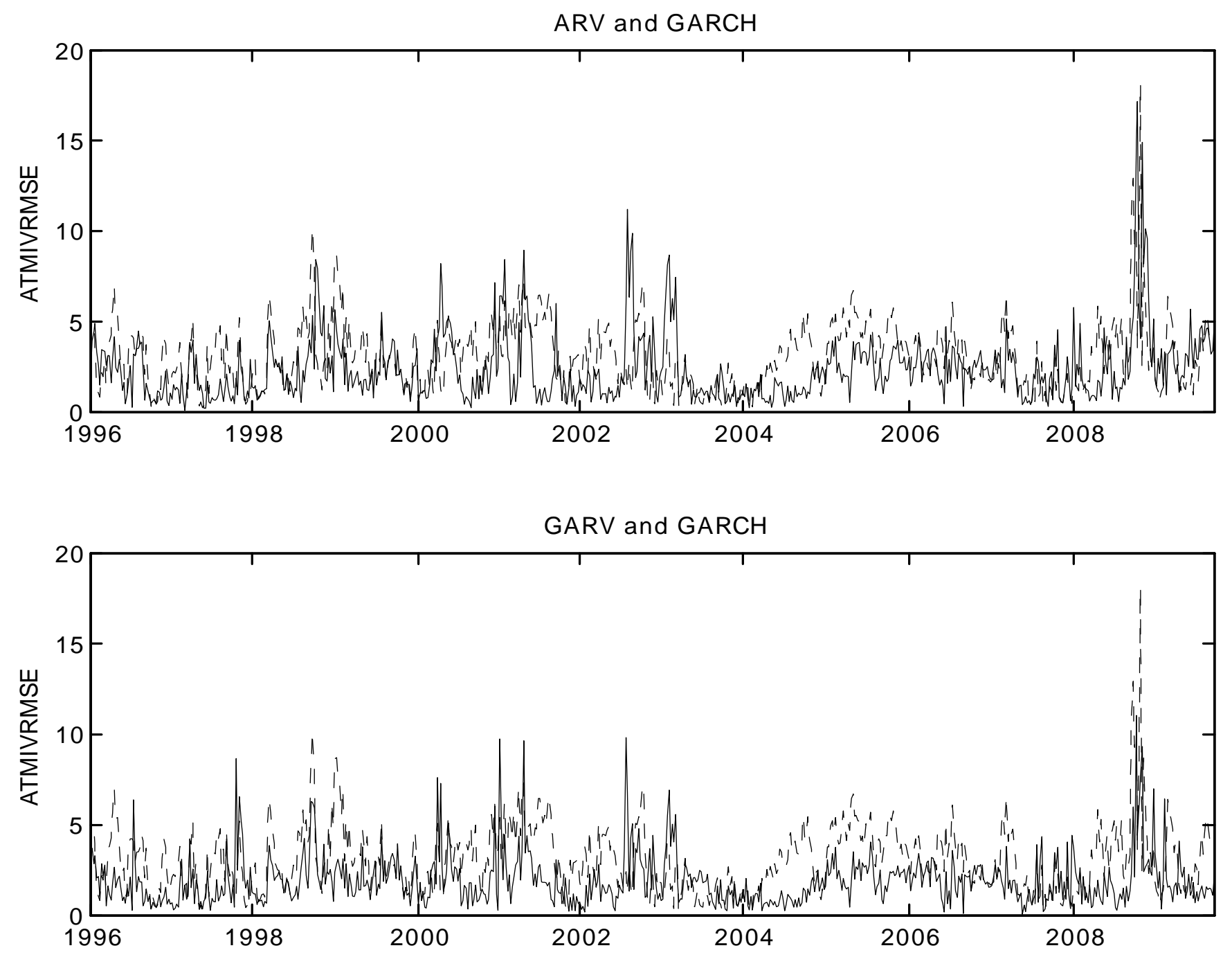

Notes to Figure: On each Wednesday we plot the implied volatility root mean squared error (IVRMSE) using at-the-money (ATM) options. The solid line in each panel corresponds to an RV-based model and the dashed line corresponds to the GARCH model. The top panel contains the ARV model and the bottom panel contains the GARV model. The model parameters are from Table 5. 
Table 1: Estimation on Daily S\&P500 Futures Returns and RV. 1990-2010.

GARCH

\section{Parameters}

$\kappa$

$\lambda$

$\alpha_{1}$

$\beta_{1}$

$\gamma_{1}$

$\omega_{1}$

$\widetilde{\alpha_{2}}$

$\widetilde{\beta_{2}}$

$\gamma_{2}$

$\widetilde{\omega_{2}}$

$\beta_{2}$

$\rho$

$E[\bar{h}]$

1

$1.30 \mathrm{E}+00$

2.83E-12

$\widetilde{a_{2}}$

$\widetilde{\beta_{2}}$

$\gamma_{2}$

$\rho$

\section{Estimate $\underline{\text { Std Error }}$}

4.03E-06 (2.70E-07)

8.29E-01 (1.19E-02)

$1.85 \mathrm{E}+02 \quad(1.24 \mathrm{E}+01)$

\section{Model Properties}

Unconditional Volatility

\section{Volam Returns}

From Returns

From RV

$\underline{\text { Log Likelihoods }}$

Returns and RV

Maximized on Returns
17.30

0.9661

1.19E-04
$(1.15 \mathrm{E}+00)$

ARV

Estimate $\underline{\text { Std Error }}$

0

$1.32 \mathrm{E}+00$

$(1.20 \mathrm{E}+00)$

$1.47 \mathrm{E}+00$

4.61E-06

9.67E-07

$4.57 \mathrm{E}+02$

5.74E-12

2.50E-06 (1.40E-06)

2.57E-06

(2.23E-07)

4.07E-06 (5.57E-01)

4.07E-06

(6.60E-02)

$6.25 \mathrm{E}+02$

$(3.52 \mathrm{E}+02)$

6.03E-12

3.30E-01

(2.92E-03)

3.43E-01

(8.40E-03)

1.03E-01

(8.77E-03)

1.03E-01

(9.50E-03)

(3.59E-06)

1.23E-04

(1.48E-05)

1.19E-04

(1.34E-05)

GARV

(3.57E-07)

(5.56E-02)

$(2.11 \mathrm{E}+01)$

$(4.54 \mathrm{E}+01)$

5.84E-12
17.58

17.37

0.3796

0.9796

0.5922

58,100

58,433

16,984

Notes: We estimate the three models using daily close-to-close returns and realized variance data for the S\&P500 index, for the period January 2, 1990 to December 31, 2010. Realized variance is constructed using the Average RV estimator. Standard errors are indicated in parentheses. The unconditional variance is estimated instead of the $\omega$ parameters which are then implied from the unconditional variance formulas. 
Table 2: S\&P500 Index Option Data. 1996-2009.

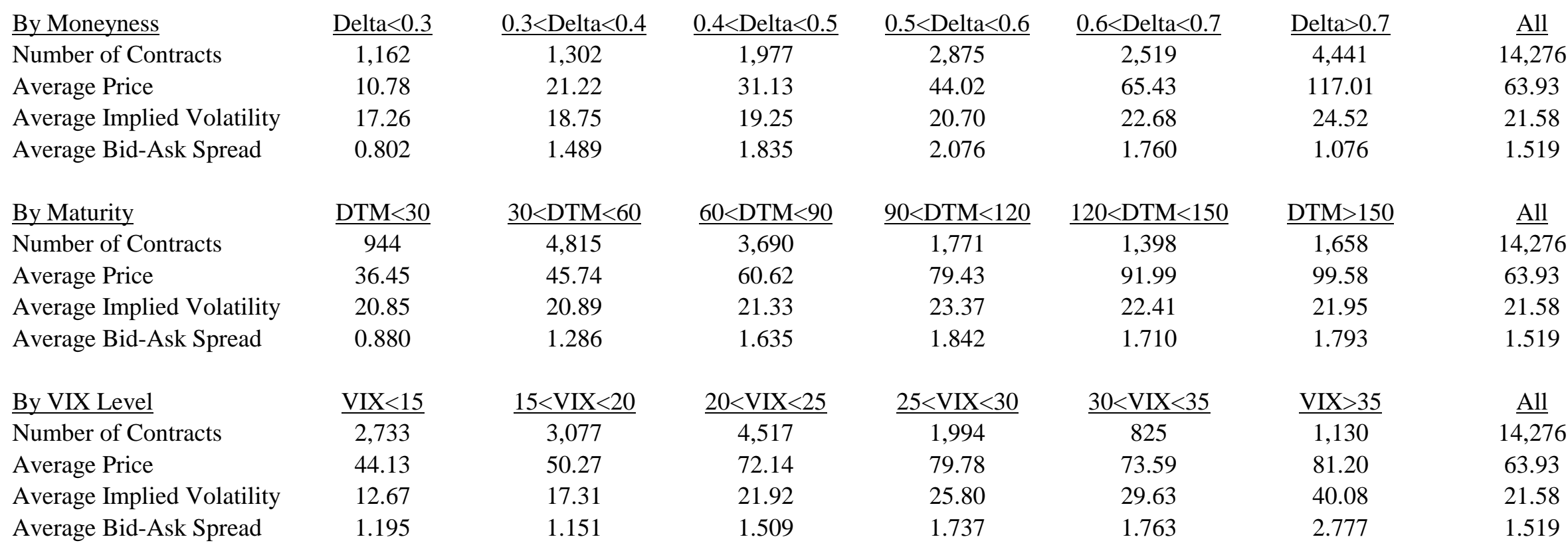

Notes: We use Wednesday closing out-of-the-money (OTM) call and put option data from OptionMetrics from January 1, 1996 through October $28,2009$.

Moneyness is measured by Delta computed from the Black-Scholes model. DTM denotes the number of calendar days to maturity. The average bid-ask spread is reported in dollars. 
Table 3: Parameter Estimation on Options. Volatility Filtered on R and RV. 1996-2009.

\begin{tabular}{|c|c|c|c|c|c|c|}
\hline \multirow[b]{2}{*}{ Parameters } & \multicolumn{2}{|c|}{ GARCH } & \multicolumn{2}{|c|}{ ARV } & \multicolumn{2}{|c|}{ GARV } \\
\hline & Estimate & Std Error & Estimate & $\underline{\text { Std Error }}$ & Estimate & Std Error \\
\hline$\kappa$ & 1 & & 0 & & $3.78 \mathrm{E}-02$ & (1.58E-03) \\
\hline$\alpha_{1}$ & 7.83E-07 & (1.77E-08) & & & 1.71E-08 & (1.47E-08) \\
\hline$\beta_{1}$ & 8.81E-01 & (3.53E-03) & & & 9.83E-01 & $(2.97 \mathrm{E}-04)$ \\
\hline$\gamma_{1}^{*}$ & $3.78 \mathrm{E}+02$ & $(9.47 \mathrm{E}+00)$ & & & $9.91 \mathrm{E}+02$ & $(4.37 \mathrm{E}+02)$ \\
\hline$\omega_{1}$ & 9.23E-15 & & & & 5.95E-14 & \\
\hline$\widetilde{\alpha_{2}}$ & & & 5.69E-07 & (2.31E-07) & 1.59E-06 & (6.97E-08) \\
\hline$\widetilde{\beta_{2}}$ & & & 4.08E-06 & (4.07E-01) & 4.08E-06 & $(4.04 \mathrm{E}-02)$ \\
\hline$\gamma_{2} *$ & & & $1.32 \mathrm{E}+03$ & $(5.38 E+02)$ & $7.85 \mathrm{E}+02$ & $(9.47 \mathrm{E}+00)$ \\
\hline$\widetilde{\omega_{2}}$ & & & 3.81E-12 & & $3.47 \mathrm{E}-12$ & \\
\hline$\beta_{2}$ & & & 1.86E-02 & (1.43E-04) & 1.53E-01 & (3.70E-03) \\
\hline$\rho$ & & & 7.25E-01 & $(2.29 \mathrm{E}-02)$ & $1.00 \mathrm{E}+00$ & (3.87E-02) \\
\hline$E^{Q}[\bar{h}]$ & $1.11 \mathrm{E}-04$ & (1.24E-06) & 7.65E-05 & $(1.21 \mathrm{E}-06)$ & 7.07E-05 & (1.17E-06) \\
\hline
\end{tabular}

\section{Model Properties}

Log Likelihood

Unconditional Volatility

Volatility Persistence

From Returns

From RV

Option Errors

IVRMSE

Ratio to GARCH

VWRMSE

Ratio to GARCH
25,486

16.75

0.9930

4.603

1.000

4.059

1.000
28,781

13.88

0.9926

3.467

0.753

3.223

0.794
30,419

13.35

0.9836

0.9406

3.145

0.683

2.873

0.708

Notes: We estimate the three models using option data for the period January 1, 1996 to October 28, 2009. Realized variance is constructed using the Average RV estimator. The unconditional variance is estimated instead of the $\omega$ parameters which are then implied from the unconditional variance formulas. Standard errors, computed using the outer product of the gradient, are indicated in parentheses. 
Table 4: IVRMSE Option Error by Moneyness, Maturity, and VIX Level. Model Parameters are Estimated on Options. Volatility Filtered on R and RV.

\begin{tabular}{|c|c|c|c|c|c|c|}
\hline \multirow[b]{2}{*}{$\underline{\text { Model }}$} & \multicolumn{6}{|c|}{ Panel A. IVRMSE by Moneyness } \\
\hline & $\underline{\text { Delta }<0.3}$ & $\underline{0.3<\text { Delta }<0.4}$ & $\underline{0.4<\text { Delta }<0.5}$ & $\underline{0.5<\text { Delta }<0.6}$ & $\underline{0.6<\text { Delta }<0.7}$ & Delta $>0.7$ \\
\hline GARCH & 4.112 & 3.590 & 3.517 & 3.772 & 4.092 & 5.974 \\
\hline ARV & 3.934 & 3.169 & 3.017 & 3.020 & 3.039 & 4.056 \\
\hline \multirow[t]{2}{*}{ GARV } & 3.451 & 2.422 & 2.456 & 2.484 & 2.749 & 4.002 \\
\hline & \multicolumn{6}{|c|}{ Panel B. IVRMSE by Maturity } \\
\hline$\underline{\text { Model }}$ & $\underline{\mathrm{DTM}<30}$ & $\underline{30<\mathrm{DTM}<60}$ & $\underline{60<\mathrm{DTM}<90}$ & $\underline{90<\mathrm{DTM}<120}$ & $\underline{120<\mathrm{DTM}<150}$ & $\underline{\mathrm{DTM}}>150$ \\
\hline GARCH & 5.425 & 4.537 & 4.299 & 4.318 & 4.883 & 4.972 \\
\hline ARV & 4.198 & 3.471 & 2.946 & 3.152 & 3.804 & 4.043 \\
\hline \multirow[t]{2}{*}{ GARV } & 3.397 & 3.083 & 2.615 & 2.937 & 3.677 & 3.892 \\
\hline & \multicolumn{6}{|c|}{ Panel C. IVRMSE by VIX Level } \\
\hline$\underline{\text { Model }}$ & $\underline{\mathrm{VIX}}<15$ & $\underline{15<\mathrm{VIX}<20}$ & $\underline{20<\mathrm{VIX}<25}$ & $\underline{25<\mathrm{VIX}<30}$ & $\underline{30<\mathrm{VIX}<35}$ & $\underline{\mathrm{VIX}}>35$ \\
\hline GARCH & 3.536 & 3.368 & 4.564 & 4.865 & 5.777 & 7.558 \\
\hline ARV & 2.431 & 2.062 & 3.512 & 4.000 & 4.960 & 5.522 \\
\hline GARV & 2.111 & 2.069 & 3.225 & 3.514 & 4.727 & 4.777 \\
\hline
\end{tabular}

Notes: We report IVRMSE option fit across moneyness, maturity, and VIX level for the models estimated in Table 3. We use Wednesday closing out-of-the-money (OTM) call and put option data from OptionMetrics from January 1, 1996 through October 28, 2009. Moneyness is measured by Delta computed from the Black-Scholes model. DTM denotes the number of calendar days to maturity. 
Table 5: Joint Estimation on Daily Returns, RVs, and Options. 1996-2009.

\begin{tabular}{ccccccc} 
& \multicolumn{2}{c}{ GARCH } & \multicolumn{2}{c}{ ARV } & \multicolumn{2}{c}{ GARV } \\
Parameters & $\underline{\text { Estimate }}$ & $\underline{\text { Std Error }}$ & $\underline{\text { Estimate }}$ & $\underline{\text { Std Error }}$ & $\underline{\text { Estimate }}$ & $\underline{\text { Std Error }}$ \\
$\kappa$ & 1 & & 0 & & $4.17 \mathrm{E}-02$ & $(4.80 \mathrm{E}-03)$ \\
$\lambda$ & $2.98 \mathrm{E}+00$ & & $6.88 \mathrm{E}+00$ & 1.1932 & $1.08 \mathrm{E}+01$ & \\
$\alpha_{1}$ & $8.09 \mathrm{E}-07$ & $(1.59 \mathrm{E}-08)$ & & & $2.20 \mathrm{E}-08$ & $(1.42 \mathrm{E}-08)$ \\
$\beta_{1}$ & $8.79 \mathrm{E}-01$ & $(2.79 \mathrm{E}-03)$ & & & $9.83 \mathrm{E}-01$ & $(2.92 \mathrm{E}-04)$ \\
$\gamma_{1}$ & $3.73 \mathrm{E}+02$ & $(7.55 \mathrm{E}+00)$ & & & $8.59 \mathrm{E}+02$ & $(2.98 \mathrm{E}+02)$ \\
$\gamma_{1}^{*}$ & $3.76 \mathrm{E}+02$ & $(7.44 \mathrm{E}+00)$ & & & $8.70 \mathrm{E}+02$ & $(2.98 \mathrm{E}+02)$ \\
$\omega_{1}$ & $1.47 \mathrm{E}-13$ & & & & $5.28 \mathrm{E}-15$ & \\
$\widetilde{\alpha_{2}}$ & & & & & & \\
$\widetilde{\beta_{2}}$ & & & $5.99 \mathrm{E}-07$ & $(2.40 \mathrm{E}-08)$ & $1.65 \mathrm{E}-06$ & $(2.21 \mathrm{E}-07)$ \\
$\gamma_{2}$ & & & $8.94 \mathrm{E}-01$ & $(3.39 \mathrm{E}-03)$ & $2.89 \mathrm{E}-06$ & $(1.21 \mathrm{E}-01)$ \\
$\gamma_{2}^{*}$ & & & $3.87 \mathrm{E}+02$ & $(1.50 \mathrm{E}+01)$ & $7.48 \mathrm{E}+02$ & $(9.89 \mathrm{E}+01)$ \\
$\widetilde{\omega_{2}}$ & & & $4.11 \mathrm{E}+02$ & $(1.50 \mathrm{E}+01)$ & $7.73 \mathrm{E}+02$ & $(9.90 \mathrm{E}+01)$ \\
$\beta_{2}$ & & & $1.66 \mathrm{E}-13$ & & $1.10 \mathrm{E}-09$ & \\
$\rho$ & & & $3.02 \mathrm{E}-02$ & $(1.98 \mathrm{E}-04)$ & $2.10 \mathrm{E}-01$ & $(1.68 \mathrm{E}-03)$ \\
$\chi$ & & & $2.53 \mathrm{E}-01$ & $(6.38 \mathrm{E}-03)$ & $4.02 \mathrm{E}-01$ & $(5.00 \mathrm{E}-03)$ \\
$E[\bar{h}]$ & & & $-2.41 \mathrm{E}+01$ & & $-2.44 \mathrm{E}+01$ & \\
& & & $3.67 \mathrm{E}-05$ & $(6.23 \mathrm{E}-07)$ & $2.23 \mathrm{E}-05$ & $(5.04 \mathrm{E}-07)$
\end{tabular}

\section{Model Properties}

Unconditional Volatility

\begin{tabular}{lccc} 
Physical & 15.03 & 9.62 & 7.49 \\
$\begin{array}{l}\text { Risk-Neutral } \\
\text { Volatility Persistence }\end{array}$ & 16.80 & 17.79 & 17.34 \\
\hline $\begin{array}{l}\text { From Returns } \\
\text { From RV }\end{array}$ & 0.9910 & & 0.9835 \\
$\begin{array}{l}\text { Log Likelihoods } \\
\text { Returns, RV and Options }\end{array}$ & & 0.9837 & 0.8871 \\
$\quad$ Returns and Options & 39,192 & & \\
Option Errors & & 73,363 & 77,285 \\
IVRMSE & 4.585 & 40,640 & 43,686 \\
$\quad$ Ratio to GARCH & 1.000 & & 3.418 \\
VWRMSE & 4.060 & 4.456 & 0.745 \\
$\quad$ Ratio to GARCH & 1.000 & 0.972 & 2.998 \\
\end{tabular}

Notes: We estimate the three models using returns, RV, and option data for the period January 1, 1996 to October 28, 2009. See the text for details. Standard errors, computed using the outer product of the gradient, are indicated in parentheses. The unconditional variance is estimated instead of the $\omega$ parameters which are then implied from the unconditional variance formulas. 
Table 6: IVRMSE Option Error by Moneyness, Maturity, and VIX Level.

Model Parameters are Estimated Jointly on Returns, RVs, and Options.

\begin{tabular}{|c|c|c|c|c|c|c|}
\hline \multirow[b]{2}{*}{$\underline{\text { Model }}$} & \multicolumn{6}{|c|}{ Panel A. IVRMSE by Delta Level } \\
\hline & $\underline{\text { Delta }<0.3}$ & $\underline{0.3<\text { Delta }<0.4}$ & $\underline{0.4<\text { Delta }<0.5}$ & $\underline{0.5}<$ Delta $<0.6$ & $\underline{0.6<\text { Delta }<0.7}$ & $\underline{\text { Delta }>0.7}$ \\
\hline GARCH & 4.137 & 3.615 & 3.537 & 3.780 & 4.082 & 5.916 \\
\hline ARV & 3.886 & 3.337 & 3.255 & 3.150 & 3.319 & 6.276 \\
\hline \multirow[t]{2}{*}{ GARV } & 3.116 & 2.497 & 2.460 & 2.446 & 2.731 & 4.731 \\
\hline & \multicolumn{6}{|c|}{ Panel B. IVRMSE by Maturity } \\
\hline$\underline{\text { Model }}$ & $\underline{\mathrm{DTM}<30}$ & $\underline{30<\mathrm{DTM}<60}$ & $\underline{60<\mathrm{DTM}<90}$ & $\underline{90<\mathrm{DTM}<120}$ & $\underline{120<\mathrm{DTM}<150}$ & $\underline{\mathrm{DTM}}>150$ \\
\hline GARCH & 5.405 & 4.527 & 4.280 & 4.267 & 4.868 & 4.967 \\
\hline ARV & 4.583 & 4.103 & 4.008 & 4.466 & 5.529 & 5.265 \\
\hline \multirow[t]{2}{*}{ GARV } & 3.799 & 3.250 & 3.020 & 3.259 & 3.948 & 4.109 \\
\hline & \multicolumn{6}{|c|}{ Panel C. IVRMSE by VIX Level } \\
\hline$\underline{\text { Model }}$ & $\underline{\mathrm{VIX}<15}$ & $\underline{15<\mathrm{VIX}<20}$ & $\underline{20<\mathrm{VIX}<25}$ & $\underline{25<\mathrm{VIX}<30}$ & $\underline{30<\mathrm{VIX}<35}$ & $\underline{\mathrm{VIX}>35}$ \\
\hline GARCH & 3.588 & 3.359 & 4.529 & 4.848 & 5.752 & 7.491 \\
\hline ARV & 2.804 & 3.087 & 4.730 & 5.395 & 6.187 & 6.147 \\
\hline GARV & 2.200 & 2.156 & 3.575 & 3.910 & 5.105 & 5.107 \\
\hline
\end{tabular}

Notes: We report IVRMSE option fit across moneyness, maturity, and VIX level for the models estimated in Table 5. We use Wednesday closing out-of-the-money (OTM) call and put option data from OptionMetrics from January 1, 1996 through October 28, 2009. Moneyness is measured by Delta computed from the Black-Scholes model. DTM denotes the number of calendar days to maturity. 
Table 7: Two-Component Models. Joint Estimation on Daily Returns, RVs, and Options. 1996-2009.

\begin{tabular}{|c|c|c|c|c|}
\hline \multirow[b]{2}{*}{ Parameters } & \multicolumn{2}{|c|}{ ARV-2C } & \multicolumn{2}{|c|}{ GARV-2C } \\
\hline & Estimate & $\underline{\text { Std Error }}$ & Estimate & $\underline{\text { Std Error }}$ \\
\hline$\kappa$ & 0 & & $4.20 \mathrm{E}-02$ & $(1.66 \mathrm{E}-02)$ \\
\hline$\lambda$ & $8.05 E+00$ & $(1.08 \mathrm{E}+00)$ & $1.07 \mathrm{E}+01$ & \\
\hline$\alpha_{1}$ & & & $2.44 \mathrm{E}-08$ & $(1.73 \mathrm{E}-08)$ \\
\hline$\beta_{1}$ & & & 9.83E-01 & $(2.92 \mathrm{E}-04)$ \\
\hline$\gamma_{1}$ & & & $8.12 \mathrm{E}+02$ & $(2.65 E+02)$ \\
\hline$\gamma_{1} *$ & & & $8.23 \mathrm{E}+02$ & $(2.65 \mathrm{E}+02)$ \\
\hline$\omega_{1}$ & & & $1.08 \mathrm{E}-10$ & \\
\hline$\alpha_{\mathrm{L}}$ & 2.87E-09 & (9.69E-11) & $1.64 \mathrm{E}-06$ & $(7.13 \mathrm{E}-06)$ \\
\hline$\beta_{\mathrm{L}}$ & $9.82 \mathrm{E}-01$ & $(2.64 \mathrm{E}-04)$ & 3.83E-03 & $(3.81 \mathrm{E}+00)$ \\
\hline$\alpha_{s}$ & $2.98 \mathrm{E}-07$ & (4.07E-09) & 7.00E-09 & (7.11E-06) \\
\hline$\beta_{\mathrm{S}}$ & 9.32E-01 & (6.29E-04) & 3.83E-03 & $(3.85 E+00)$ \\
\hline$\gamma_{2}$ & $1.77 \mathrm{E}+03$ & $(1.35 \mathrm{E}+01)$ & $7.49 \mathrm{E}+02$ & $(1.13 E+02)$ \\
\hline$\gamma_{2}{ }^{*}$ & $1.79 \mathrm{E}+03$ & $(1.38 \mathrm{E}+01)$ & $7.73 \mathrm{E}+02$ & $(1.13 E+02)$ \\
\hline$\omega_{2}$ & 2.98E-07 & & 7.19E-09 & \\
\hline$\left(\alpha_{L}+\alpha_{S}\right) / \alpha_{2}$ & $9.25 \mathrm{E}-02$ & (7.73E-04) & 2.10E-01 & $(2.72 \mathrm{E}-03)$ \\
\hline$\rho$ & 3.18E-01 & (3.83E-03) & 4.01E-01 & (5.03E-03) \\
\hline$\chi$ & $-1.99 \mathrm{E}+01$ & & $-2.44 \mathrm{E}+01$ & \\
\hline$E[\bar{h}]$ & $3.42 \mathrm{E}-05$ & (4.48E-07) & 2.23E-05 & $(7.04 \mathrm{E}-07)$ \\
\hline
\end{tabular}

\section{Model Properties}

Unconditional Volatility

Physical 9.28

Risk-Neutral 15.69

7.50

17.34

Volatility Persistence

From Returns LRC

From Returns SRC

From RV LRC

From RV SRC

Log Likelihoods

Returns, RV and Options

Returns and Options

Option Errors

IVRMSE

Ratio to GARCH

VWRMSE

15.69

0.9835

0.9912

0.8870

0.9320

0.0038

Ratio to GARCH

75,940

77,286

42,456

43,685

3.973

3.418

0.867

0.745

3.268

2.998

0.805

0.738

Notes: We estimate the three models using returns, RV, and option data for the period January 1, 1996 to October 28, 2009. The unconditional variance is estimated instead of the $\omega$ parameters which are then implied from the unconditional variance formulas. Standard errors, computed using the outer product of the gradient, are indicated in parentheses. 
Table 8: Non-Affine Models. Estimation on Daily Returns and RV. 1990-2010.

\begin{tabular}{|c|c|c|c|c|c|c|}
\hline \multirow{3}{*}{$\begin{array}{c}\text { Parameters } \\
\kappa\end{array}$} & \multicolumn{2}{|c|}{ NGARCH } & \multicolumn{2}{|c|}{ NARV } & \multicolumn{2}{|c|}{ GNARV } \\
\hline & Estimate & $\underline{\text { Std Error }}$ & Estimate & Std Error & Estimate & $\underline{\text { Std Error }}$ \\
\hline & 1 & & 0 & & $6.21 \mathrm{E}-01$ & (2.39E-02) \\
\hline$\lambda$ & 8.31E-01 & (9.70E-01) & $1.45 \mathrm{E}+00$ & $(1.19 \mathrm{E}+00)$ & $3.15 E+00$ & (4.53E-01) \\
\hline$\alpha_{1}$ & $6.70 \mathrm{E}-02$ & $(4.54 \mathrm{E}-03)$ & & & 3.22E-02 & $(2.39 \mathrm{E}-03)$ \\
\hline$\beta_{1}$ & 8.49E-01 & (7.58E-03) & & & $6.55 \mathrm{E}-07$ & $(5.05 \mathrm{E}-02)$ \\
\hline$\gamma_{1}$ & $1.04 \mathrm{E}+00$ & (8.05E-02) & & & $5.48 \mathrm{E}+00$ & (3.36E-01) \\
\hline$\omega_{1}$ & $1.80 \mathrm{E}-06$ & & & & 4.91E-11 & \\
\hline$\widetilde{\alpha_{2}}$ & & & 9.58E-02 & (1.16E-03) & 8.10E-02 & (6.19E-03) \\
\hline$\widetilde{\beta_{2}}$ & & & $5.78 \mathrm{E}-01$ & (3.89E-03) & $8.10 \mathrm{E}-02$ & (2.88E-02) \\
\hline$\gamma_{2}$ & & & $1.83 \mathrm{E}+00$ & (2.56E-03) & $2.33 \mathrm{E}+00$ & (1.70E-01) \\
\hline$\widetilde{\omega_{2}}$ & & & 1.73E-06 & & 4.74E-06 & \\
\hline$\beta_{2}$ & & & 4.80E-01 & (3.61E-03) & 5.31E-01 & (1.10E-02) \\
\hline$\rho$ & & & 1.45E-01 & (2.64E-03) & 1.44E-01 & (9.76E-03) \\
\hline$E[\bar{h}]$ & 1.67E-04 & $(3.40 \mathrm{E}-05)$ & 2.49E-04 & (9.94E-05) & $1.68 \mathrm{E}-04$ & (3.47E-05) \\
\hline
\end{tabular}

Model Properties

Unconditional Volatility

20.53

25.07

20.56

Volatility Persistence

From Returns

0.9887

0.6407

From RV

0.8973

0.6879

Log Likelihoods

Returns and RV

59,682

60,065

Maximized on Returns $\quad 16,931$

16,984

17,042

Notes: We estimate the three models using daily close-to-close returns and realized variance data for the S\&P500 index, for the period January 2, 1990 to December 31, 2010. Realized variance is constructed using the Average RV estimator. Standard errors are indicated in parentheses. The unconditional variance is estimated instead of the $\omega$ parameters which are then implied from the unconditional variance formulas. 